\title{
NET CONVERGENCE STRUCTURES WITH APPLICATIONS TO VECTOR LATTICES
}

\author{
M. O'BRIEN, V.G. TROITSKY, AND J.H. VAN DER WALT
}

\begin{abstract}
Convergence is a fundamental topic in analysis that is most commonly modelled using topology. However, there are many natural convergences that are not given by any topology; e.g., convergence almost everywhere of a sequence of measurable functions and order convergence of nets in vector lattices. The theory of convergence structures provides a framework for studying more general modes of convergence. It also has one particularly striking feature: it is formalized using the language of filters. This paper develops a general theory of convergence in terms of nets. We show that it is equivalent to the filter-based theory and present some translations between the two areas. In particular, we provide a characterization of pretopological convergence structures in terms of nets. We also use our results to unify certain topics in vector lattices with general convergence theory.
\end{abstract}

\section{INTRODUCTION}

Convergence is an important part of the toolkit for anyone working in functional analysis, where many deep properties are often expressed in terms of convergent nets and sequences. While convergence is often associated with topology, it has long been known that there are important convergences that are not topological: it was shown in Ord66 that convergence almost everywhere is not given by a topology. Furthermore, there are several important non-topological convergences in the theory of vector lattices, including order convergence, uo-convergence, and relative uniform convergence.

The theory of convergence structures was developed in order to handle non-topological convergences. For an overview of this subject area

Date: March 3, 2021.

The second author was supported by an NSERC grant. 
we refer the reader to the monograph BB02]. Of particular note, this theory uses the language of filters to describe convergence; we will refer to this approach as the theory of filter convergence structures. In this paper, we develop a theory of net convergence structures that is equivalent to filter convergence theory. As the name suggests, our approach will focus on describing convergence using the language of nets. While several authors have approached this idea, including [Kel55, p. 73], [Kat67, AA72, Ars77, Pea88, [Sch97, p. 168-170], and [HZW10, AEG], our paper has several advantages. First and foremost, our theory is equivalent to the theory of filter convergence structures despite the use of nets. As a consequence, all results from the theory of filter convergence structures remain applicable to net convergence structures. Our definition also handles several set-theoretic subtleties, like how the collection of all nets in a given set does not form a set.

This paper can be broken into two main parts: the general theory of net convergence structures, which is developed in Sections 2, 8, and applications to vector lattices in Sections 9 and 10. Section 2 covers preliminaries and set-theoretic considerations, while the definition of net convergence structures and their basic properties are presented in Section 3. We provide a brief overview of filter convergence structures in Section 4, and show they are equivalent to our theory of net convergence in Section 5. Section [6 is a dictionary between several basic concepts from the filter theory and the language of nets. A reader not interested in filter convergence structures may skip Sections 4, 5, and 6: in a way, the entire point of this paper is that the filter theory is not required to study convergence structures. We characterize topological and pretopological convergence structures in terms of nets in Section 7. In Section 8 we reinvestigate continuous convergence from the perspective of nets. In the final two sections we discuss applications of our theory to vector lattices: Section 9 focuses on order convergence from the point of view of convergence structures, while we identify the Mackey and topological modifications of order convergence in Section 10.

For results concerning topological vector spaces, we refer the reader to [KN76] and Section 3.1 in [AB06]. 


\section{What EXACTLY IS A NET?}

The definition of a net is not consistent throughout the literature: some authors require the index set to be ordered, while others allow pre-ordered index sets. Some authors may require index sets to be infinite and without terminal nodes. We will take the most general approach.

It is a common in the literature to quantify over all nets in a given set. For example, the standard definition of an order closed set in a vector lattice goes as follows: a set $C$ is said to be order closed if for every net $\left(x_{\alpha}\right)$ in $C$, if the net converges in order to $x$, it implies $x \in C$. This definition is "illegal" from the point of view of ZFC: we quantify over all nets in $C$, hence we implicitly quantify over all directed sets, which is not a set. One way to resolve this issue is to use NBG rather than ZFC and consider classes of nets; this is the approach taken in [Kel55, p. 73]. We describe another way to circumvent this issue while staying within ZFC.

Let $A$ be a set equipped with a relation " $\leqslant$ ". A relation is a preorder if it is reflexive and transitive. Note that $x \sim y$ if $x \leqslant y$ and $y \leqslant x$ then defines an equivalence relation. A pre-order may be easily converted into an order when necessary; this allows one to view any pre-ordered set as an ordered set. One way to do this is to replace each equivalence class with a single point to produce a "quotient order". Another approach is to "straighten up" each equivalence class by using the Well Ordering Principle to redefine the relation on each equivalence class to make it into a totally ordered set.

A set $A$ is directed if it is pre-ordered and every two elements have a common descendant; more formally

$$
\forall \alpha, \beta \in A \quad \exists \gamma \in A \text { such that } \alpha \leqslant \gamma \text { and } \beta \leqslant \gamma
$$

Throughout this section $X$ will denote an arbitrary set. A function from a directed set $A$ to $X$ is referred to as a net in $X$ indexed by $A$. In this case, instead of writing $x: A \rightarrow X$ we write $\left(x_{\alpha}\right)_{\alpha \in A}$ or just $\left(x_{\alpha}\right)$ if the index set is clear from the context. A sequence is simply a net indexed by $\mathbb{N}$. 
Let $\left(x_{\alpha}\right)_{\alpha \in A}$ be a net in $X$. We write $\left\{x_{\alpha}\right\}_{\alpha \in A}$ for the set of all terms of the net: $\left\{x_{\alpha}: \alpha \in A\right\}$, which is nothing but the range of $x$ viewed as a function. If $\alpha_{0} \in A$ is fixed and we put $A_{0}=\left\{\alpha \in A: \alpha \geqslant \alpha_{0}\right\}$ then $A_{0}$ is again a directed set under the pre-order induced from $A$. The restriction of the function $x$ to $A_{0}$ is called a tail of $\left(x_{\alpha}\right)$, and it is denoted by $\left(x_{\alpha}\right)_{\alpha \geqslant \alpha_{0}}$. The range of this new net will be denoted by $\left\{x_{\alpha}\right\}_{\alpha \geqslant \alpha_{0}}$ and called a tail set of $\left(x_{\alpha}\right)_{\alpha \in A}$. By $\left\langle x_{\alpha}\right\rangle_{\alpha \in A}$ we mean the set of all tail sets of $\left(x_{\alpha}\right)_{\alpha \in A}$; that is,

$$
\left\langle x_{\alpha}\right\rangle_{\alpha \in A}=\left\{\left\{x_{\alpha}\right\}_{\alpha \geqslant \alpha_{0}}: \alpha_{0} \in A\right\} .
$$

Convergence is often thought of as a "tail property", meaning that altering terms at the "head" of a convergent net should not affect convergence. So, intuitively, convergence should be determined by tail sets. This idea is embedded into the following two definitions. Following Kat67], given two nets $\left(x_{\alpha}\right)_{\alpha \in A}$ and $\left(y_{\beta}\right)_{\beta \in B}$ we say that $\left(y_{\beta}\right)_{\beta \in B}$ is a quasi-subnet of $\left(x_{\alpha}\right)_{\alpha \in A}$ and write $\left(y_{\beta}\right)_{\beta \in B} \preceq\left(x_{\alpha}\right)_{\alpha \in A}$ if for every $\alpha_{0} \in A$ there exists $\beta_{0} \in B$ such that $\left\{y_{\beta}\right\}_{\beta \geqslant \beta_{0}} \subseteq\left\{x_{\alpha}\right\}_{\alpha \geqslant \alpha_{0}}$; this just means every tail of $\left(x_{\alpha}\right)_{\alpha \in A}$ contains a tail of $\left(y_{\beta}\right)_{\beta \in B}$ as a subset.

If both $\left(y_{\beta}\right)_{\beta \in B} \preceq\left(x_{\alpha}\right)_{\alpha \in A}$ and $\left(x_{\alpha}\right)_{\alpha \in A} \preceq\left(y_{\beta}\right)_{\beta \in B}$, we say that $\left(x_{\alpha}\right)_{\alpha \in A}$ and $\left(y_{\beta}\right)_{\beta \in B}$ are tail equivalent and write $\left(x_{\alpha}\right)_{\alpha \in A} \sim\left(y_{\beta}\right)_{\beta \in B}$. While this relation will be sufficient for our main results, we frequently observe a stronger property. $\left(x_{\alpha}\right)_{\alpha \in A}$ and $\left(y_{\beta}\right)_{\beta \in B}$ are said to be stongly tail equivalent, written $\left(x_{\alpha}\right)_{\alpha \in A} \approx\left(y_{\beta}\right)_{\beta \in B}$, if $\left\langle x_{\alpha}\right\rangle_{\alpha \in A}=\left\langle y_{\beta}\right\rangle_{\beta \in B}$.

It is easy to see that " $\preceq$ " is reflexive and transitive, while " $\sim$ " and " $\approx$ " are reflexive, transitive, and symmetric. While it is tempting to say " $\preceq$ " is a pre-order and " " and " $\approx$ " are equivalence relations on the nets on $X$, the latter collection is not a set. The following technical trick will allow us to work around this issue.

Our idea is to restrict the collection of index sets to a set that is large enough to represent any possible tail behaviour in $X$. We write $\mathcal{P}(X)$ for the power set of $X$ and define $V_{1}(X)=X$ and $V_{n+1}(X)=V_{n}(X) \cup$ $\mathcal{P}\left(V_{n}(X)\right)$ for $n \in \mathbb{N}$; this is formally known as the superstructure over $X$. A net $\left(x_{\alpha}\right)_{\alpha \in A}$ in $X$ is called admissible if $A \in \bigcup_{n=1}^{\infty} V_{n}(X)$.

\footnotetext{
${ }^{1}$ also see AA72
} 
Unlike all nets in $X$, the admissible nets clearly form a set; we denote it by $\mathfrak{N}(X)$. It is easy to see that the restriction of " $\preceq$ " to $\mathfrak{N}(X)$ is a pre-order relation, and the restrictions of " $\sim$ " and " $\approx$ " are equivalence relations.

We will delay the proof of this next theorem until Section 5 where it will be deduced as an easy corollary.

Theorem 2.1. Every net in $X$ is strongly tail equivalent to an admissible net.

Since any reasonable definition of convergence for nets should be stable under tail equivalence, this theorem effectively says we may, without loss of generality, restrict our attention to admissible nets. In view of this, we will often identify an arbitrary net $\left(x_{\alpha}\right)_{\alpha \in A}$ in $X$ with the equivalence class in $\mathfrak{N}(X) / \sim$ consisting of the admissible nets that are tail equivalent to $\left(x_{\alpha}\right)_{\alpha \in A}$.

There are several non-equivalent definitions of the term subnet in the literature and, for historical reasons, we would like to mention the two that are most common. Keep in mind that, in the end, the exact definition of subnet will be of little concern to us because our theory will be based on quasi-subnets. Let $\left(x_{\alpha}\right)_{\alpha \in A}$ be a net, $B$ be a directed set, and $\varphi: B \rightarrow A$ such that Range $\varphi$ is co-final in $A$, meaning for every $\alpha_{0} \in A$ there is $\beta_{0} \in B$ with $\varphi(\beta) \geqslant \alpha_{0}$ whenever $\beta \geqslant \beta_{0}$. The composition $x \circ \varphi: B \rightarrow X$ is a net in $X$ indexed by $B$ and is called a subnet of $\left(x_{\alpha}\right)_{\alpha \in A}$ according to Kelley in [Kel55]. Willard in Wil70] requires the additional assumption that $\varphi$ be monotone. Clearly every Willard-subnet is a Kelley-subnet. We refer the reader to [Sch97, pp. 162-165] for a thorough discussion about several alternative definitions of a subnet and relationships between these definitions. The following result is essentially contained in Corollary 7.19 in Sch97.

Proposition 2.2. Every subnet is a quasi-subnet. Every quasi-subnet is tail-equivalent to a subnet.

Proof. It is easy to see that every subnet (either Willard or Kelley) is a quasi-subnet. Let $\left(y_{\beta}\right)_{\beta \in B}$ be a quasi-subnet of $\left(x_{\alpha}\right)_{\alpha \in A}$. Put $C=\left\{(\alpha, \beta) \in A \times B: x_{\alpha}=y_{\beta}\right\}$. For $(\alpha, \beta) \in C$, put $\varphi(\alpha, \beta)=\alpha$. It 
is straightforward that $C$ is directed under the product order induced from $A \times B$, and $x \circ \varphi$ is a subnet of $\left(x_{\alpha}\right)_{\alpha \in A}$ (in the sense of both Willard and Kelley), which is tail equivalent to $\left(y_{\beta}\right)_{\beta \in B}$.

Example 2.3. (i) The sequences $(1,0,0,0, \ldots)$ and $(-1,0,0,0, \ldots)$ are tail equivalent, but not stronlgy tail equivalent. In particular, each is a quasi-subnet of the other. Notice that neither is a subnet of the other in the sense of either Kelley or Willard.

(ii) Let $\left(x_{n}\right)_{n \in \mathbb{N}}$ and $\left(y_{n}\right)_{n \in \mathbb{N}}$ be sequences in $\mathbb{N}$ given by

$$
\begin{aligned}
\left(x_{n}\right)_{n \in \mathbb{N}} & =(1,2,3,4,5,6,7,8, \ldots) \\
\text { and } \quad\left(y_{n}\right)_{n \in \mathbb{N}} & =(2,1,4,3,6,5,8,7, \ldots) .
\end{aligned}
$$

It is easy to see that these two sequences are tail equivalent. Note that $\left(x_{n}\right)_{n \in \mathbb{N}}$ is increasing while $\left(y_{n}\right)_{n \in \mathbb{N}}$ is not. So the property of being monotone is not preserved under tail equivalence. Also note that the two sequences are not strongly tail equivalent. However, each is a Kelley-subnet of the other via $\varphi(2 n-1)=2 n$ and $\varphi(2 n)=2 n-1(n \in \mathbb{N})$.

(iii) The sequences $(-1,0,1,2,3, \ldots)$ and $(0,-1,0,1,2,3, \ldots)$ are strongly tail equivalent, yet one is increasing while the other is not: so monotonicity is not preserved under strong tail equivalence.

A net $\left(x_{\alpha}\right)$ in a partially ordered set is said to be increasing if $x_{\alpha} \leqslant x_{\beta}$ in $X$ whenever $\alpha \leqslant \beta$ in $A$. One defines decreasing nets similarly. The preceding example shows that tail equivalence may ruin important properties of nets, like monotonicity. This has the potential to cause serious problems for applications to vector lattices. There is, however, a fix for this issue.

Proposition 2.4. Let $X$ be a partially ordered set, and let $\left(x_{\alpha}\right)_{\alpha \in \Lambda}$ be an increasing net in $X$. Then there exists an admissible increasing net in $X$ which is strongly tail equivalent to $\left(x_{\alpha}\right)_{\alpha \in \Lambda}$. A similar result holds for decreasing nets. 
Proof. For each $\alpha \in \Lambda$ let $T_{\alpha}=\left\{x_{\beta}\right\}_{\beta \geqslant \alpha}$ be the $\alpha$-th tail set of the original net. It is clear that $x_{\alpha}$ is the (unique) least element of $T_{\alpha}$. Let

$$
\mathcal{B}=\left\langle x_{\alpha}\right\rangle_{\alpha \in \Lambda}=\left\{T_{\alpha}: \alpha \in \Lambda\right\}
$$

and order it by reverse inclusion. This makes $\mathcal{B}$ into a partially ordered directed set. Define a net $\left(y_{A}\right)_{A \in \mathcal{B}}$ by letting $y_{A}$ be the least element of $A$. It can be easily verified that this net is increasing, and it is admissible because $\mathcal{B} \in \mathcal{P}(\mathcal{P}(X))$.

Let $\alpha_{0} \in \Lambda$. If $A \geqslant T_{\alpha_{0}}$ in $\mathcal{B}$ then $y_{A} \in A \subseteq T_{\alpha_{0}}$, which implies $\left\{y_{A}\right\}_{A \geqslant T_{\alpha_{0}}} \subseteq T_{\alpha_{0}}$. Furthermore, for every $\alpha \geqslant \alpha_{0}$ in $\Lambda$ we have $T_{\alpha} \geqslant T_{\alpha_{0}}$ in $\mathcal{B}$ and $x_{\alpha}=y_{T_{\alpha}}$; hence $T_{\alpha} \subseteq\left\{y_{A}\right\}_{A \geqslant T_{\alpha_{0}}}$. This yields $\left\{y_{A}\right\}_{A \geqslant T_{\alpha_{0}}}=$ $T_{\alpha_{0}}$, and thus the two nets have the same tail sets. Therefore, they are strongly tail equivalent.

The case of decreasing nets may be handled in a similar fashion.

This proposition motivates the following definition. Let $X$ be a partially ordered set. An equivalence class in $\mathfrak{N}(X) / \sim$ is said to be $\boldsymbol{i n}$ creasing if it contains an increasing net and decreasing if it contains a decreasing net.

Remark 2.5. When dealing with a pair of nets, the following trick allows one to assume the two nets have the same index set. Let $\left(x_{\alpha}\right)_{\alpha \in A}$ and $\left(y_{\beta}\right)_{\beta \in B}$ be two nets. The Cartesian product $A \times B$ can be made into a directed set by defining $\left(\alpha_{1}, \beta_{1}\right) \leqslant\left(\alpha_{2}, \beta_{2}\right)$ whenever $\alpha_{1} \leqslant \alpha_{2}$ in $A$ and $\beta_{1} \leqslant \beta_{2}$ in $B$. Now define two new nets indexed by $A \times B$ via

$$
x_{(\alpha, \beta)}^{\prime}=x_{\alpha} \quad \text { and } \quad y_{(\alpha, \beta)}^{\prime}=y_{\beta}, \quad \text { where } \quad(\alpha, \beta) \in A \times B .
$$

It is easy to see that $\left(x_{\alpha}\right)_{\alpha \in A}$ is strongy tail equivalent to $\left(x_{(\alpha, \beta)}^{\prime}\right)_{(\alpha, \beta) \in A \times B}$ and $\left(y_{\beta}\right)_{\beta \in B}$ is strongly tail equivalent to $\left(y_{(\alpha, \beta)}^{\prime}\right)_{(\alpha, \beta) \in A \times B}$.

\section{Net CONVERGENCE STRUCTURES}

We are now in a position to introduce the main object of this paper. Fix a set $X$. By a net convergence structure on $X$ we mean a function $\eta: X \rightarrow \mathcal{P}((\mathfrak{N}(X))$ satisfying certain axioms that will be given below. Instead of $\left(x_{\alpha}\right)_{\alpha \in \Lambda} \in \eta(x)$, we write $\left(x_{\alpha}\right)_{\alpha \in \Lambda} \stackrel{\eta}{\rightarrow} x$ and say that $\left(x_{\alpha}\right)_{\alpha \in \Lambda} \eta$-converges to $x$. For convenience, and when there is 
no risk of confusion, we may de-emphasize $\eta$ and simply write $x_{\alpha} \rightarrow x$ and say $\left(x_{\alpha}\right)$ converges to $x$. In all these cases, $x$ is called a limit of $\left(x_{\alpha}\right)_{\alpha \in \Lambda}$. Here are the axioms:

(N1) Constant nets converge: if $x_{\alpha}=x$ for every $\alpha$ then $x_{\alpha} \rightarrow x$;

(N2) If a net converges to $x$ then every quasi-subnet of it converges to $x$;

(N3) Suppose that $\left(x_{\alpha}\right)_{\alpha \in \Lambda} \rightarrow x$ and $\left(y_{\alpha}\right)_{\alpha \in \Lambda} \rightarrow x$. Let $\left(z_{\alpha}\right)_{\alpha \in \Lambda}$ be a net in $X$ such that $z_{\alpha} \in\left\{x_{\alpha}, y_{\alpha}\right\}$ for every $\alpha$. Then $z_{\alpha} \rightarrow x$.

In this case, we say that $X$ together with this net convergence structure is a net convergence space. If we wish to highlight both the set and the convergence on it, we will usually write $(X, \stackrel{\eta}{\rightarrow})$ or $(X, \rightarrow)$.

If $x_{\alpha} \rightarrow x$ and $\left(x_{\alpha}\right) \sim\left(y_{\beta}\right)$ for some admissible nets $\left(x_{\alpha}\right)$ and $\left(y_{\beta}\right)$, then $y_{\beta} \rightarrow x$; this follows immediately from the fact that $\left(y_{\beta}\right)$ is a quasi-subnet of $\left(x_{\alpha}\right)$. In particular, an admissible net converges to $x$ iff some (and therefore every) tail of it converges to $x$. This also means that one may think of convergence of equivalence classes in $\mathfrak{N}(X) / \sim$ rather than just for individual nets.

We now extend convergence to nets that need not be admissible: for an arbitrary net $\left(x_{\alpha}\right)$ in $X$ we say $x_{\alpha} \rightarrow x$ if $\left(x_{\alpha}\right)$ is tail equivalent to an admissible net that converges to $x$. However, every time we quantify over "all nets in $X$ ", we implicitly mean "over $\mathfrak{N}(X)$ ".

Given two convergence structures $\eta_{1}$ and $\eta_{2}$ on $X$, we say that $\eta_{1}$ is stronger than $\eta_{2}$ if $x_{\alpha} \stackrel{\eta_{1}}{\rightarrow} x$ implies $x_{\alpha} \stackrel{\eta_{2}}{\rightarrow} x$ for every (admissible) net $\left(x_{\alpha}\right)$ in $X$; in this case we also say that $\eta_{2}$ is weaker than $\eta_{1}$.

Every convergence induced by a topology satisfies the axioms above; such convergences will be referred to as topological. We may now extend many properties of convergence from topology to the setting of general net convergence spaces.

A net convergence space is said to be Hausdorff if every net has at most one limit. If the structure is induced by a topology, this just means the topology is Hausdorff.

It follows from Proposition 2.2 that the axiom (N2) is equivalent to the following two alternative conditions:

(N2a) If a net converges to $x$, then so does each of its subnets, and 
(N2b) If $\left(x_{\alpha}\right)$ converges to $x$ and $\left(y_{\beta}\right)$ is tail equivalent to $\left(x_{\alpha}\right)$ then $\left(y_{\beta}\right)$ converges to $x$.

Example 3.1. The following example shows that (N1) and (N2) do not imply (N3), even in the Hausdorff case. For a net $\left(x_{\alpha}\right)$ in $\mathbb{R}$, we define $x_{\alpha} \rightarrow x$ if $\left(x_{\alpha}\right)$ converges to $x$ in the usual convergence of $\mathbb{R}$ and, in addition, $\left(x_{\alpha}\right)$ either has a tail consisting of only rational numbers or only of irrational numbers. It is easy to see that this convergence satisfies (N1) and (N2) and is Hausdorff, yet it fails (N3).

Example 3.2. There are important convergences that fail to form net convergence structures. For example, convergence along an ultrafilter fails (N2).

Let $X$ be a net convergence space and $A \subseteq X$. We denote the set of limits of all nets in $A$ by $\bar{A}$ and call it the closure or the adherence of $A$. If the limit of every convergent net in $A$ belongs to $A$, we say that $A$ is closed and write $\bar{A}=A$. A word of warning: the closure of a set need not be closed! We say that $A$ is dense if $\bar{A}=X$. For $x \in A \subseteq X$ we say that $A$ is a neighborhood of $x$ if for every net in $X$ that converges to $x$, a tail of the net is contained in $A$. $A$ is said to be open if it is a neighborhood of each of its points. A function $f: X \rightarrow Y$ between two net convergence spaces is said to be continuous at $x$ if $x_{\alpha} \rightarrow x$ in $X$ implies $f\left(x_{\alpha}\right) \rightarrow f(x)$ in $Y$ for every admissible net $\left(x_{\alpha}\right)$ in $X$. Note that the net $\left(f\left(x_{\alpha}\right)\right)$ need not be admissible in $Y$; it suffices that it is tail equivalent to an admissible net in $Y$ that converges to $f(x)$. We say that $f$ is continuous if it is continuous at every $x \in X$.

Let $\mathcal{A}$ be a family of functions from a set $X$ to a collection of net convergence spaces. We now consider the net convergence structure on $X$ induced by $\mathcal{A}$ : put $x_{\alpha} \rightarrow x$ whenever $f\left(x_{\alpha}\right) \rightarrow f(x)$ for every $f \in \mathcal{A}$. It can be easily verified that this is the weakest net convergence structure that makes every function in $\mathcal{A}$ continuous.

Let $X$ be a net convergence space and $Y \subseteq X$. There is a natural way to define a net convergence structure on $Y$ : for a net $\left(x_{\alpha}\right)$ in $Y$ and a point $x$ in $Y$ we put $x_{\alpha} \rightarrow x$ in $Y$ whenever $x_{\alpha} \rightarrow x$ in the original convergence on $X$. We call $Y$ a subspace of $X$. Equivalently, 
this is the convergence structure on $Y$ induced by the inclusion map of $Y$ into $X$; i.e., it is the weakest net convergence structure on $Y$ that makes the inclusion into $X$ continuous.

$X$ is said to be compact if every net in $X$ has a convergent quasisubnet. Then we say that a subset $A$ of $X$ is compact if it is compact with respect to the induced convergence structure; equivalently, if every net in $A$ has a quasi-subnet that converges in $X$ to an element of $A$. Several features of compact subsets from topology continue to hold in the setting of net convergence spaces. For example, every compact subspace of a Hausdorff space is closed. It is also easy to see that every real- or complex-valued continuous function is bounded on every compact subset of $X$. A net convergence space is locally compact if every convergent net has a tail contained in a compact set.

Let $\left(X_{\gamma}\right)_{\gamma \in \Gamma}$ be a family of net convergence spaces and consider the Cartesian product $X=\prod_{\gamma \in \Gamma} X_{\gamma}$. For every $\gamma \in \Gamma$ let $P_{\gamma}: X \rightarrow X_{\gamma}$ be the canonical coordinate map. The product net convergence structure on $X$ is given by $x_{\alpha} \rightarrow x$ in $X$ whenever $P_{\gamma} x_{\alpha} \rightarrow P_{\gamma} x$ in $X_{\gamma}$ for every $\gamma$. Equivalently, this is the net convergence structure on $X$ induced by the family of all coordinate maps $\left\{P_{\gamma}\right\}_{\gamma \in \Gamma}$; we view this as the "point-wise" or "coordinate-wise" convergence.

All the concepts introduced in the preceding paragraphs agree with the appropriate topological concepts when the convergence is topological. We list several of these properties in the following proposition, all of which have well-known analogues for filter convergence structures. In Section 5 we will prove that our concept of a net convergence structure is equivalent to the concept of a filter convergence structure; this will make Proposition 3.3 redundant. Nevertheless, we provide a proof of the proposition in order to illustrate the simplicity of net convergence techniques.

Proposition 3.3. The following are valid in every net convergence structure:

(i) The composition of two continuous functions is continuous;

(ii) Every set is contained in its closure;

(iii) A set is open iff its complement is closed; 
(iv) The intersection of any collection of closed sets is closed;

(v) The union of any finite collection of closed sets is closed;

(vi) The union of any collection of open sets is open;

(vii) The intersection of any finite collection of open sets is open.

Proof. (ii), (ii), (iv) are straightforward.

To prove (iii), first suppose that $A$ is open. Let $\left(x_{\alpha}\right)$ be a net in $A^{C}$ such that $x_{\alpha} \rightarrow x$, yet $x \in A$. Then a tail of the net is in $A$, which is a contradiction.

Conversely, if $A^{C}$ is closed, $\left(x_{\alpha}\right)_{\alpha \in \Lambda}$ converges to $x \in A$, and no tail of the net is contained in $A$, then let $\Lambda_{0}=\left\{\alpha \in \Lambda: x_{\alpha} \in A^{C}\right\}$. It is easy to see that $\Lambda$ is directed and the restriction of $\left(x_{\alpha}\right)_{\alpha \in \Lambda}$ to $\Lambda_{0}$ is a quasi-subnet of $\left(x_{\alpha}\right)_{\alpha \in \Lambda}$. This quasi-subnet is contained in $A^{C}$ and still converges to $x$ by (N2). Since this implies $x \in A^{C}$, we have reached a contradiction.

(vi) follows from (iii) and (iv).

(vii) It suffices to prove the statements for two sets and apply induction. Let $A_{1}$ and $A_{2}$ be two open sets; we need to show that $A_{1} \cap A_{2}$ is open. Suppose that $x \in A_{1} \cap A_{2}$ and $x_{\alpha} \rightarrow x$. Since $x \in A_{1}$ and $A_{1}$ is open, there exists $\alpha_{1}$ such that the tail set $\left\{x_{\alpha}\right\}_{\alpha \geqslant \alpha_{1}}$ is contained in $A_{1}$. Similarly, some tail set $\left\{x_{\alpha}\right\}_{\alpha \geqslant \alpha_{2}}$ is contained in $A_{2}$. Now choose any $\alpha_{0} \geqslant \alpha_{1}, \alpha_{2}$ and note that the tail set $\left\{x_{\alpha}\right\}_{\alpha \geqslant \alpha_{0}}$ is contained in $A_{1} \cap A_{2}$.

(v) follows from (iii) and (vii).

Even though the terminology resembles that from topology, note that there are important differences. In particular, in a convergence structure, the closure of a set need not be closed:

Example 3.4. The following example is derived from [BB02]. Let $X=\mathbb{R}$ and define a convergence $x_{\alpha} \rightarrow x$ as follows: if $x \neq 0$ put $x_{\alpha} \rightarrow x$ whenever $\left(x_{\alpha}\right)$ converges to $x$ in the usual topology of $\mathbb{R}$; otherwise, define $x_{\alpha} \rightarrow 0$ whenever $\left(x_{\alpha}\right)$ converges to 0 in the usual topology of $\mathbb{R}$ and a tail of the net is contained in $\mathbb{Q}$. Observe that $\overline{\mathbb{R} \backslash \mathbb{Q}}=\mathbb{R} \backslash\{0\}$. The latter set is not closed since its closure is $\mathbb{R}$. In particular, this shows that changing the convergence at a single point is enough to spoil a topological convergence. 
There are several natural ways in which a given net convergence structure induces another convergence. We call the new structure a modification of the original one. Let $X$ be a net convergence space. It follows from Proposition 3.3 that the collection of all open subsets of $X$ forms a topology, say, $\tau$. We call $\tau$ the topology associated with the convergence structure. This topology induces its own convergence, which we will denote $x_{\alpha} \stackrel{\tau}{\rightarrow} x$; it is called the topological modification of the original convergence structure.

Proposition 3.5. Let $(X, \rightarrow)$ be a convergence space and $\tau$ its topological modification. Then $x_{\alpha} \rightarrow x$ implies $x_{\alpha} \stackrel{\tau}{\rightarrow} x$. Moreover, $\tau$ is the finest topology on $X$ whose convergence is weaker than the original convergence.

Proof. If $x_{\alpha} \rightarrow x$ then, by the definition of an open set, every open neighborhood of $x$ contains a tail of $\left(x_{\alpha}\right)$ and hence $x_{\alpha} \stackrel{\tau}{\rightarrow} x$.

Let $\sigma$ be a topology on $X$ such that $x_{\alpha} \rightarrow x$ implies $x_{\alpha} \stackrel{\sigma}{\rightarrow} x$. Let $A$ be a $\sigma$-closed set. If $\left(x_{\alpha}\right)$ is a net in $A$ and $x_{\alpha} \rightarrow x$, then $x_{\alpha} \stackrel{\sigma}{\rightarrow} x$; so $x \in A$. It follows that $A$ is closed in $(X, \rightarrow)$, so it is $\tau$-closed.

It follows that the topological modification is generally weaker than the original convergence. We will see in Example 10.7 that it may be dramatically weaker. Clearly, a net convergence structure is topological precisely when it equals its topological modification.

Net convergence vector spaces. Throughout the rest of the section, let $X$ be a vector space over $\mathbb{K}$, where $\mathbb{K}$ is either $\mathbb{R}$ or $\mathbb{C}$, equipped with the usual convergence. A net convergence structure on $X$ is said to be linear if addition and scalar multiplication operations are jointly continuous. We then say that $X$ is a net convergence vector space. It is easy to see that $x_{\alpha} \rightarrow x$ iff $x_{\alpha}-x \rightarrow 0$.

A linear operator $T: X \rightarrow Y$ between two net convergence vector spaces is said to be an isomorphism if $T$ is a bijection and both $T$ and $T^{-1}$ are continuous. We say that $T$ is an isomorphic embedding if $T$ is an isomorphism when viewed as a map from $X$ to Range $T$.

Definition 3.6. A subset $A$ of net convergence vector space $X$ is said to be bounded if $\left(r_{\alpha} x\right)_{(\alpha, x)} \rightarrow 0$ whenever $\left(r_{\alpha}\right)_{\alpha \in \Lambda} \rightarrow 0$ in $\mathbb{K}$. In this 
definition $\left(r_{\alpha} x\right)_{(\alpha, x)}$ is interpreted as a net over the index set $\Lambda \times A$ directed via $(\alpha, x) \leqslant(\beta, y)$ whenever $\alpha \leqslant \beta$. With a minor abuse of notation, we will denote this net by $\left(r_{\alpha} A\right)$. A net convergence vector space is said to be locally bounded if every convergent net has a bounded tail.

Proposition 3.7. Let $A$ and $B$ be subsets of a convergence vector space $X$.

(i) If $A$ is bounded and $B \subseteq A$ then $B$ is bounded;

(ii) If $A$ is bounded then $r A$ is bounded for every $r \geqslant 0$;

(iii) If $A$ and $B$ are both bounded then so are $A \cup B$ and $A+B$;

(iv) Every finite set is bounded.

Proof. The proofs of (ii) and (iii) are straightforward. To prove (iii) we let $A$ and $B$ be two bounded sets in $X, C=A \cup B$, and $\left(r_{\alpha}\right)_{\alpha \in \Lambda} \rightarrow 0$ in $\mathbb{K}$. We may assume without loss of generality that both $A$ and $B$ are non-empty. As before, we view $\Lambda \times A, \Lambda \times B$, and $\Lambda \times C$ as directed sets under $(\alpha, x) \leqslant(\beta, y)$ whenever $\alpha \leqslant \beta$. Fix any $a_{0} \in A$ and $b_{0} \in B$. Define two nets, one in $A$ and the other in $B$, both indexed by $\Lambda \times C$ as follows: for $(\alpha, c)$ in $\Lambda \times C$, put

$$
x_{(\alpha, c)}=\left\{\begin{array}{ll}
r_{\alpha} c & \text { if } c \in A \\
r_{\alpha} a_{0} & \text { otherwise }
\end{array} \quad y_{(\alpha, c)}= \begin{cases}r_{\alpha} c & \text { if } c \in B \\
r_{\alpha} b_{0} & \text { otherwise. }\end{cases}\right.
$$

Observe that $\left(x_{(\alpha, c)}\right)_{(\alpha, c) \in \Lambda \times C}$ is strongly tail equivalent to the net $\left(r_{\alpha} A\right)$. Since $A$ is bounded, the latter net converges to zero and hence, $x_{(\alpha, c)} \rightarrow$ 0 . Similarly, we have $y_{(\alpha, c)} \rightarrow 0$. Observe that for every $(\alpha, c)$ in $\Lambda \times C$ we have

$$
r_{\alpha} c= \begin{cases}x_{(\alpha, c)} & \text { if } c \in A \\ y_{(\alpha, c)} & \text { if } c \in B .\end{cases}
$$

Axiom (N3) yields $\left(r_{\alpha} c\right)_{(\alpha, c) \in \Lambda \times C} \rightarrow 0$, which shows $C$ is bounded.

Let $D=A+B$. For every $d \in D$, choose $a \in A$ and $b \in B$ such that $d=a+b$, and put $a=f(d)$ and $b=g(d)$. Define two nets indexed by $\Lambda \times D$ as follows: $x_{(\alpha, d)}=r_{\alpha} f(d)$ and $y_{(\alpha, d)}=r_{\alpha} g(d)$. It is easy to see $\left(x_{\alpha, d}\right)_{\Lambda \times D} \preceq\left(r_{\alpha} A\right)$ and $\left(y_{\alpha, d}\right)_{\Lambda \times D} \preceq\left(r_{\alpha} B\right)$ hence $x_{\alpha, d} \rightarrow 0$ and 
$y_{\alpha, d} \rightarrow 0$. It follows that $r_{\alpha} d=x_{\alpha, d}+y_{\alpha, d} \rightarrow 0$ and, as a consequence, $D$ is bounded.

(iv) It follows from the continuity of scalar multiplication that every singleton is bounded; now apply (iii).

Recall that the circled hull of a set $A$ is the set $\{r x:|r| \leqslant 1, x \in$ $A\}$. We say that $A$ is circled if it equals its circled hull or, equivalently, $x \in A$ implies $r x \in A$ whenever $|r| \leqslant 1$. Note that if $A$ is bounded then so is its circled hull. While this fact can be verified directly, it will be almost trivial in the filter language: see page 17. It is also easy to see that if $A$ is circled then the net $\left(r_{\alpha}\right)$ in Definition 3.6 may be taken to be the sequence $\left(\frac{1}{n}\right)_{n \in \mathbb{N}}$. That is, a circled set $A$ is bounded iff $\frac{1}{n} A \rightarrow 0$. We also make use of the fact that a bounded set $A$ has a bounded convex hull: the latter is contained in $C+C$ where $C$ is the circled hull of $A$.

A net $\left(x_{\alpha}\right)_{\alpha \in \Lambda}$ in a net convergence vector space is said to be $\boldsymbol{C a u c h y}$ if the net $\left(x_{\alpha}-x_{\beta}\right)_{(\alpha, \beta) \in \Lambda \times \Lambda}$ converges to zero. Here $\Lambda \times \Lambda$ is directed coordinate-wise. It is easy to see that every convergent net is Cauchy. Indeed, put $y_{(\alpha, \beta)}=x_{\alpha}$ and $z_{(\alpha, \beta)}=x_{\beta}$. The nets $\left(y_{(\alpha, \beta)}\right)$ and $\left(z_{(\alpha, \beta)}\right)$ are both strongly tail equivalent to $\left(x_{\alpha}\right)$, so they both converge to $x$. It follows that $x_{\alpha}-x_{\beta}=y_{(\alpha, \beta)}-z_{(\alpha, \beta)} \rightarrow 0$. A net convergence vector space is said to be complete if every Cauchy net is convergent.

Let $X$ be a topological vector space. In this case, our definitions of boundedness, Cauchy and completeness agree with the standard ones given in [KN76, pp. 44 and 56]. In fact, for the terms Cauchy and completeness, our definitions are exactly the same. As for boundedness, according to [KN76, p. 44], a subset $A$ of a topological vector space is bounded if for every neighborhood of zero $U$ there exists a $t>0$ such that $A \subseteq t U$. It is easy to see that this agrees with our Definition 3.6.

Let $(X, \rightarrow)$ be a Hausdorff net convergence vector space. We define a new net convergence structure on $X$ as follows: for a net $\left(x_{\alpha}\right)$, we write $x_{\alpha} \stackrel{\mathrm{m}}{\rightarrow} x$ if there exists a bounded set $B$ such that for every $\varepsilon>0$ there is an $\alpha_{0}$ such that $x_{\alpha}-x \in \varepsilon B$ whenever $\alpha \geqslant \alpha_{0}$. It can be easily verified that $(X, \stackrel{\mathrm{m}}{\rightarrow})$ is indeed a linear net convergence structure on $X$; it is called the Mackey modification of the original structure. 
Proposition 3.8. If $x_{\alpha} \stackrel{\mathrm{m}}{\rightarrow} x$ then $x_{\alpha} \rightarrow x$.

Proof. Since both structures are linear, we may assume that $x=0$. Let $B$ be a bounded set that witnesses $x_{\alpha} \stackrel{\mathrm{m}}{\rightarrow} 0$ as above. The net $\left(\frac{1}{n} B\right)$ as in Definition 3.6 satisfies $\frac{1}{n} b \rightarrow 0$. Now observe that $\left(x_{\alpha}\right)$ is a quasi-subnet of $\left(\frac{1}{n} B\right)$.

Proposition 3.9. Let $T: X \rightarrow Y$ be a linear map between net convergence vector spaces $X$ and $Y$. The following are equivalent:

(i) $T$ is bounded; that is, $T$ maps bounded sets to bounded sets;

(ii) $T$ is Mackey-continuous: $x_{\alpha} \stackrel{\mathrm{m}}{\rightarrow} x$ implies $T x_{\alpha} \stackrel{\mathrm{m}}{\rightarrow} T x$;

(iii) $x_{\alpha} \stackrel{\mathrm{m}}{\rightarrow} x$ implies $T x_{\alpha} \rightarrow T x$.

Proof. (ii) $\Rightarrow$ (iii) is straightforward; (iii) $\Rightarrow$ (iii) follows from the preceding proposition.

(iii) $\Rightarrow$ (ii) Suppose that $B$ is a bounded subset of $X$. It remains to show that $T(B)$ is bounded. We may assume that $B$, and therefore $T(B)$, is circled. Let $\left(r_{\alpha}\right)_{\alpha \in \Lambda} \rightarrow 0$ in $\mathbb{R}$. Since $B$ is bounded, we have $\left(r_{\alpha} b\right) \stackrel{\mathrm{m}}{\rightarrow} 0$ where $\left(r_{\alpha} b\right)$ is indexed by $\Lambda \times B$ directed by the first component. It follows that $\left(r_{\alpha} T b\right) \rightarrow 0$, so $T(B)$ is bounded.

A linear convergence structure is Mackey if it agrees with its Mackey modification.

\section{Filter convergence structures}

This section contains a brief review of terminology from filter convergence structures; we refer the reader to [BB02] for a detailed exposition.

We begin by fixing some notation. For a set $X$, a non-empty collection $\mathcal{B}$ of non-empty subsets of $X$ is said to be a filter base if it is directed with respect to reverse inclusion: for all $A, B \in \mathcal{B}$ there exists $C \in \mathcal{B}$ such that $C \subseteq A \cap B$. A non-empty collection $\mathcal{F}$ of non-empty subsets of $X$ is said to be a filter if it is closed under taking supersets and finite intersections. It is easy to see that every filter is a filter base. For a filter base $\mathcal{B}$, we use $[\mathcal{B}]$ to denote the collection of all supersets of sets in $\mathcal{B}$; this forms a filter and is called the filter generated by $\mathcal{B}$. We write $\mathfrak{F}(X)$ for the set of all filters on $X$. 
A filter $\mathcal{U}$ is said to be an ultrafilter if it is not properly contained in another filter. Equivalently, for every $A \subseteq X$, either $A \in \mathcal{U}$ or $A^{C} \in \mathcal{U}$. It follows from Zorn's Lemma that every filter is contained in an ultrafilter. Any singleton $\{x\}$ is clearly a filer base; in this case $[\{x\}]$ is denoted by $[x]$. It is easy to verify this is an ultrafilter.

Let $X$ be a set and $\lambda$ be a map from $X$ to $\mathcal{P}(\mathfrak{F}(X))$. We write $\mathcal{F} \stackrel{\lambda}{\rightarrow} x$ instead of $\mathcal{F} \in \lambda(x)$, or $\mathcal{F} \rightarrow x$ when there is no risk of confusion, and say that $\mathcal{F}$ converges to $x$. In any case $x$ is called a limit of $\mathcal{F}$. We say that $\lambda$ is a filter convergence structure if the following axioms are satisfied:

$(\mathrm{F} 1)[x] \rightarrow x$ for every $x \in X$;

(F2) If $\mathcal{F} \rightarrow x$ and $\mathcal{F} \subseteq \mathcal{G}$ then $\mathcal{G} \rightarrow x$;

(F3) If $\mathcal{F} \rightarrow x$ and $\mathcal{G} \rightarrow x$ then $\mathcal{F} \cap \mathcal{G} \rightarrow x$.

A set equipped with a filter convergence structure is called a filter convergence space.

Every topology on $X$ gives rise to a natural filter convergence structure. It is easy to see that for every $x \in X$ the collection of all neighborhoods of $x$ forms a filter; we will denote it by $\mathcal{N}_{x}$. Consider a filter $\mathcal{F}$ convergent to $x$ if $\mathcal{N}_{x} \subseteq \mathcal{F}$. It is easy to see that this defines a filter convergence structure on $X$; any filter convergence structure that arises in this way is called topological.

Let $X$ be a filter convergence space. We say that $X$ is Hausdorff if every filter has at most one limit. For $x \in X$, the filter $\mathcal{U}_{x}=\bigcap\{\mathcal{F}$ : $\mathcal{F} \rightarrow x\}$ is called the neighborhood filter of $x$, and any set $U \in \mathcal{U}_{x}$ is called a neighborhood of $x$. A subset $A$ of $X$ is open if it is a neighborhood of each of its points. Again, given $A \subseteq X$, we define

$$
\bar{A}=\{x \in X: \mathcal{F} \rightarrow x \text { for some filter } \mathcal{F} \text { such that } A \in \mathcal{F}\} .
$$

We call $\bar{A}$ the closure or the adherence of $A$. Naturally, we say that $A$ is closed if $\bar{A}=A$ and dense if $\bar{A}=X$. It can be verified that $A$ is open iff $A^{C}$ is closed; see Lemma 1.3.4 in [BB02]. It can also be easily verified that the collection of all open sets in $X$ form a topology. As outlined above, this topology induces a natural filter convergence structure on $X$; we call it the topological modification of the original filter convergence structure. 
Let $f: X \rightarrow Y$ be a function between two filter convergence spaces. For every filter $\mathcal{F}$ on $X$, the collection $\{f(A): A \in \mathcal{F}\}$ is a filter base in $Y$; the filter that it generates is called the image filter of $\mathcal{F}$ under $f$ and is denoted by $f(\mathcal{F})$. We say that $f$ is continuous at $x$ if $\mathcal{F} \rightarrow x$ implies $f(\mathcal{F}) \rightarrow f(x)$.

Let $\mathcal{A}$ be a family of functions from a set $X$ to filter convergence spaces. For every $x \in X$, put $\mathcal{F} \rightarrow x$ in $X$ whenever $f(\mathcal{F}) \rightarrow f(x)$ for every $f \in \mathcal{A}$. This is the weakest filter convergence structure that makes all $f \in \mathcal{A}$ continuous. This allows us to define the subspace filter convergence structure on a subset of a filter convergence space as the weakest structure that makes the inclusion map continuous. Likewise, we define the product filter convergence structure on the Cartesian product of filter convergent spaces as the weakest structure that makes all the coordinate projections continuous.

A filter convergence space $X$ is said to be compact if every ultrafilter converges. A subset $A$ of $C$ is said to be compact if it is compact in the subspace filter convergence structure induced by $X$. We say that $X$ is locally compact if every convergent filter on $X$ contains a compact set.

A filter convergence space is first countable at $x$ if $\mathcal{F} \rightarrow x$ implies that there exists a filter $\mathcal{G}$ with a countable base such that $\mathcal{G} \subseteq \mathcal{F}$ and $\mathcal{G} \rightarrow x$. A filter convergence space is said to be first countable if it is first countable at every point.

We now take $X$ to be a vector space over $\mathbb{K}$. A filter convergence structure on $X$ is said to be linear if the operations of addition and scalar multiplication are jointly continuous; here $\mathbb{K}$ is equipped with the filter convergence structure induced by the usual topology of $\mathbb{K}$. We then call $X$ a filter convergence vector space.

Let $X$ be a filter convergence vector space and $A \subseteq X$. Let $\mathcal{N}_{0}$ denote the filter of all neighborhoods of zero in $\mathbb{K}$ and put $\mathcal{N}_{0} A=$ $\left\{U A: U \in \mathcal{N}_{0}\right\}$. It is easy to see that $\mathcal{N}_{0} A$ is a filter base. $A$ is said to be a bounded set if $\left[\mathcal{N}_{0} A\right] \rightarrow 0$ in $X$. The space $X$ is locally bounded if every convergent filter contains a bounded set. Let $A$ be a bounded set and use $C$ to denote its circled hull. It is easy to see that $\mathcal{N}_{0} A=\mathcal{N}_{0} C$, so $C$ is also bounded. 
Let $X$ be a filter convergence vector space and $\mathcal{F}$ a filter on $X$. The collection $\{A-B: A, B \in \mathcal{F}\}$ is a filter base; we write $\mathcal{F}-\mathcal{F}$ for the filter generated by this collection. $\mathcal{F}$ is called Cauchy if $\mathcal{F}-\mathcal{F} \rightarrow 0$ and we say that $X$ is complete if every Cauchy filter on $X$ converges.

For a filter $\mathcal{F}$ on a filter convergence vector space $X$, define $\mathcal{F} \stackrel{\mathrm{m}}{\rightarrow} 0$ if $\mathcal{F} \supseteq \mathcal{N}_{0} B$ for some bounded set $B$. It follows that $\mathcal{F} \stackrel{\mathrm{m}}{\rightarrow} x$ if $\mathcal{F}-x \stackrel{\mathrm{m}}{\rightarrow}$ 0 defines a new linear filter convergence structure on $X$, called the Mackey modification of the original structure.

\section{Equivalence of Net AND Filter struCtures}

We now show that the concepts of net and filter convergence structures are equivalent. Throughout this section $X$ denotes an arbitrary set, unless specified otherwise.

Observe that for a net $\left(x_{\alpha}\right)$, its collection of tail sets $\left\langle x_{\alpha}\right\rangle$ is a filter base; we will call it the tail filter base of $\left(x_{\alpha}\right)$. The filter generated by this filter base will be called the tail filter of $\left(x_{\alpha}\right)$ and denoted by $\left[x_{\alpha}\right]$. The definition of strong tail equivalence between two nets means they have the same tail filter bases. This is a stronger property than two nets being tail equivalent, which can be characterized in terms of tail filters: $\left(x_{\alpha}\right) \preceq\left(y_{\beta}\right)$ iff $\left[y_{\beta}\right] \subseteq\left[x_{\alpha}\right]$. Thus, two nets are tail equivalent iff their tail filters agree.

Proposition 5.1. Every filter base on $X$ is the tail filter base of some admissible net in $X$.

Proof. Let $\mathcal{B}$ be a filter base on $X$. Let $\Lambda$ be the subset of $\mathcal{B} \times X$ defined by $(A, x) \in \Lambda$ whenever $x \in A$ :

$$
\Lambda=\{(A, x): x \in A \in \mathcal{B}\}
$$

A pre-order can be defined on $\Lambda$ via $(A, x) \leqslant(B, y)$ when $B \subseteq A$; this makes $\Lambda$ a directed set. Define a net indexed by $\Lambda$ via $x_{(A, x)}=$ $x$. It is easy to see that this net is admissible and its tail filter base equals $\mathcal{B}$.

We are now ready to prove Theorem 2.1 
Proof of Theorem 2.1. Let $\left(x_{\alpha}\right)$ be a net in $X$. By Proposition 5.1. there is an admissible net whose tail filter base is $\left\langle x_{\alpha}\right\rangle$, hence this new net is strongly tail equivalent to $\left(x_{\alpha}\right)$.

Proposition 5.1 implies that there is a natural bijection between $\mathfrak{N}(X) / \sim$ and $\mathfrak{F}(X)$. Indeed, it follows from Proposition 5.1 that the map induced by associating every net in $\mathfrak{N}(X)$ to its tail filter is a map from $\mathfrak{N}(X)$ onto $\mathfrak{F}(X)$. By the definition of tail equivalence, this map induces a bijection from $\mathfrak{N}(X) / \sim$ to $\mathfrak{F}(X)$.

The next two theorems show that the bijection between $\mathfrak{N}(X) / \sim$ and $\mathfrak{F}(X)$ induces a bijection between the set of all net convergence structures and the set of all filter convergence structures on $X$. This will help us show that Section 3 and Section 4 are exact translations of each other.

Theorem 5.2. Let $X$ be a net convergence space. Then $X$ is also a filter convergence space under the filter convergence structure given by $\mathcal{F} \rightarrow x$ whenever $\mathcal{F}$ is the tail filter of some net converging to $x$.

Proof. We need to verify (F1)-(F3). (F1) is trivial because, for every $x \in X,[x]$ is the tail filter of any constant net in $X$ with constant value $x$, and such a net converges to $x$ by (N1).

To show (F2), suppose $\mathcal{F} \rightarrow x$ and $\mathcal{F} \subseteq \mathcal{G}$. It follows from $\mathcal{F} \rightarrow x$ that $\mathcal{F}=\left[x_{\alpha}\right]$ for some net $\left(x_{\alpha}\right)$ where $x_{\alpha} \rightarrow x$. Proposition 5.1 allows us to find a net $\left(y_{\beta}\right)$ in $\mathfrak{N}(X)$ such that $\mathcal{G}=\left[y_{\beta}\right]$. Now $\mathcal{F} \subseteq \mathcal{G}$ implies $\left(y_{\beta}\right) \preceq\left(x_{\alpha}\right)$. Therefore, $y_{\beta} \rightarrow x$ by (N2), and hence $\mathcal{G} \rightarrow x$.

Finally, we prove (F3). Assume $\mathcal{F} \rightarrow x$ and $\mathcal{G} \rightarrow x$. Then we can find two nets $\left(x_{\alpha}\right)_{\alpha \in \Lambda}$ and $\left(y_{\alpha}\right)_{\alpha \in \Lambda}$ in $\mathfrak{N}(X)$ satisfying $\mathcal{F}=\left[x_{\alpha}\right], \mathcal{G}=\left[y_{\alpha}\right]$, $x_{\alpha} \rightarrow x$, and $y_{\alpha} \rightarrow x$; note that we may assume the two nets have the same index set by Remark 2.5. Letting $\Gamma=\Lambda \times\{1,2\}$ and defining $(\alpha, i) \leqslant(\beta, j)$ in $\Gamma$ if $\alpha \leqslant \beta$ in $\Lambda$ makes $\Gamma$ into a directed set. Define a net $\left(z_{\gamma}\right)_{\gamma \in \Gamma}$ by setting $z_{(\alpha, 1)}=x_{\alpha}$ and $z_{(\alpha, 2)}=y_{\alpha}$. It is easy to see that both $\left(x_{\alpha}\right)$ and $\left(y_{\alpha}\right)$ are quasi-subnets of $\left(z_{\gamma}\right)$ and, as a consequence, $\left[z_{\gamma}\right] \subseteq\left[x_{\alpha}\right] \cap\left[y_{\alpha}\right]=\mathcal{F} \cap \mathcal{G}$. It now suffices to verify $z_{\gamma} \rightarrow x$ because this would imply $\left[z_{\gamma}\right] \rightarrow x$ and, therefore, $\mathcal{F} \cap \mathcal{G} \rightarrow x$ by (F2).

Define two auxiliary nets $\left(\bar{x}_{\gamma}\right)_{\gamma \in \Gamma}$ and $\left(\bar{y}_{\gamma}\right)_{\gamma \in \Gamma}$ as follows: put $\bar{x}_{(\alpha, i)}=$ $x_{\alpha}$ and $\bar{y}_{(\alpha, i)}=y_{\alpha}$. It is easy to see $\left(\bar{x}_{\gamma}\right)$ and $\left(\bar{y}_{\gamma}\right)$ are strongly tail 
equivalent to $\left(x_{\alpha}\right)$ and $\left(y_{\alpha}\right)$, respectively, so $\bar{x}_{\gamma} \rightarrow x$ and $\bar{y}_{\gamma} \rightarrow x$. Since $z_{(\alpha, 1)}=\bar{x}_{(\alpha, 1)}$ and $z_{(\alpha, 2)}=\bar{y}_{(\alpha, 2)}$ for every $\alpha$, (N3) yields $z_{\gamma} \rightarrow z$.

Theorem 5.3. Let $X$ be a filter convergence space. Then it is also a net convergence space under the net convergence structure given by $x_{\alpha} \rightarrow x$ whenever $\left[x_{\alpha}\right] \rightarrow x$.

Proof. It is easy to see that axiom (F1) of the original filter convergence implies (N1) for the new net convergence. To verify (N2), suppose $x_{\alpha} \rightarrow$ $x$ and $\left(y_{\beta}\right)$ is a quasi-subnet of $\left(x_{\alpha}\right)$. Then $\left[x_{\alpha}\right] \rightarrow x$ and $\left[x_{\alpha}\right] \subseteq\left[y_{\beta}\right]$ implies $\left[y_{\beta}\right] \rightarrow x$ by (F2), which yields $y_{\beta} \rightarrow x$.

To show (N3), we assume $x_{\alpha} \rightarrow x, y_{\alpha} \rightarrow x$, and let $\left(z_{\alpha}\right)$ be such that $z_{\alpha} \in\left\{x_{\alpha}, y_{\alpha}\right\}$ for every $\alpha$. Let $A \in\left[x_{\alpha}\right] \cap\left[y_{\alpha}\right]$. It follows that $A$ contains a tail of $\left(x_{\alpha}\right)$ and a tail of $\left(y_{\alpha}\right)$. Then there exists $\alpha_{0}$ such that both $\left\{x_{\alpha}\right\}_{\alpha \geqslant \alpha_{0}}$ and $\left\{y_{\alpha}\right\}_{\alpha \geqslant \alpha_{0}}$ are subsets of $A$. This shows $\left\{z_{\alpha}\right\}_{\alpha \geqslant \alpha_{0}}$ is a subset of $A$, so $A \in\left[z_{\alpha}\right]$. It follows that $\left[x_{\alpha}\right] \cap\left[y_{\alpha}\right] \subseteq\left[z_{\alpha}\right]$. Since $\left[x_{\alpha}\right] \rightarrow x$ and $\left[y_{\alpha}\right] \rightarrow x,\left(\right.$ F3) implies $\left[x_{\alpha}\right] \cap\left[y_{\alpha}\right] \rightarrow x$. Now (F2) yields $\left[z_{\alpha}\right] \rightarrow x$, which implies $z_{\alpha} \rightarrow x$.

Theorem 5.2 yields a map from net convergence structures on $X$ to filter convergence structures on $X$, while Theorem 5.3 yields a map in the opposite direction. Thus, every net convergence structure gives rise to an associated filter convergence structure, and every filter convergence structure gives rise to an associated net convergence structure. It is easy to verify that these two maps are inverses of each other. Going forward, we will use the term convergence structure instead of net convergence structure or filter convergence structure. Similarly, we will now talk about convergence spaces and convergence vector spaces. We will see that the net and filter languages each have their own advantages and disadvantages, so one should use the language that is most convenient in a given situation.

In [HZW10], the authors consider a definition of net convergence structure consisting of just two axioms that are analogous to our axioms (N1) and (N2). However, their definition does not include (N3). They observe that their definition is implied by filter convergence structures and ask whether the two approaches are equivalent. We now know that 
they are not: (N3) is needed to ensure the equivalence: Example 3.1 shows that (N1) and (N2) are not sufficient.

\section{Equivalence of Net AND Filter terminologies}

In Sections 3 and 4 we introduced various terms in the settings of net and filter convergence structures. We will now show that translating between the net and filter languages preserves the meaning of all these terms. For example, a set in a net convergence vector space $X$ is bounded iff it is bounded in the associated filter convergence structure on $X$.

Throughout this section we take $X$ to be a convergence space. In order to distinguish between properties, we will use prefixes "net" and "filter". For example, we write "net-bounded" versus "filter-bounded" to distinguish boundedness in the two settings. As before, "net" will usually mean "admissible net" when quantifying over all nets.

Most of these results are easy exercises, but we present their proofs for the convenience of the reader.

Hausdorff: Suppose $X$ is filter-Hausdorff, and assume $x_{\alpha} \rightarrow x$ and $x_{\alpha} \rightarrow y$. Then $\left[x_{\alpha}\right] \rightarrow x$ and $\left[x_{\alpha}\right] \rightarrow y$ implies $x=y$; hence, $X$ is netHausdorff. Conversely, if $X$ is net-Hausdorff and $\mathcal{F} \rightarrow x$ and $\mathcal{F} \rightarrow y$, find a net $\left(x_{\alpha}\right)$ such that $\mathcal{F}=\left[x_{\alpha}\right]$. It follows that $x_{\alpha} \rightarrow x$ and $x_{\alpha} \rightarrow y$, so $x=y$, confirming that $X$ is filter-Hausdorff.

Continuous functions: Let $f: X \rightarrow Y$ be a function between two convergence spaces. It is easy to see $f\left(\left[x_{\alpha}\right]\right)=\left[f\left(x_{\alpha}\right)\right]$ for every net $\left(x_{\alpha}\right)$ in $X$. If $x_{\alpha} \rightarrow x$ in $X$ and $f$ is filter-continuous, then $\left[x_{\alpha}\right] \rightarrow$ $x$ and $\left[f\left(x_{\alpha}\right)\right]=f\left(\left[x_{\alpha}\right]\right) \rightarrow f(x)$. These imply $f\left(x_{\alpha}\right) \rightarrow f(x)$ and, consequently, that $f$ is net continuous. Suppose now that $f$ is net continuous and $\mathcal{F} \rightarrow x$ in $X$. Find a net $\left(x_{\alpha}\right)$ in $X$ such that $x_{\alpha} \rightarrow x$ and $\mathcal{F}=\left[x_{\alpha}\right]$. It follows that $f\left(x_{\alpha}\right) \rightarrow f(x)$, so $f(\mathcal{F})=\left[f\left(x_{\alpha}\right)\right] \rightarrow$ $f(x)$, and $f$ is filter-continuous.

All the properties that were defined using continuous functions are then the same in net and filter structures. These include subspaces, product spaces, and convergence vector spaces. 
Closure: Let $x$ belong to the net-closure of $A$. Then there is a net $\left(x_{\alpha}\right)$ in $A$ such that $x_{\alpha} \rightarrow x$. Then $\left[x_{\alpha}\right] \rightarrow x$. It follows from $A \in\left[x_{\alpha}\right]$ that $x$ is in the filter-closure of $A$. Conversely, suppose that $x$ is in the filter-closure of $A$. Then $A \in \mathcal{F} \rightarrow x$ for some filter $\mathcal{F}$. It follows that there is a net $\left(x_{\alpha}\right)$ such that $\mathcal{F}=\left[x_{\alpha}\right]$ and $x_{\alpha} \rightarrow x$. Then $A \in\left[x_{\alpha}\right]$ implies $A$ contains a tail of $\left(x_{\alpha}\right)$. Since this tail still converges to $x$, we conclude that $x$ is in the net-closure of $A$.

It now follows that the concepts of closed, open, and dense sets, as well as topological modification, agree in net and filter structures.

Neighborhoods: we leave it as an exercise that net-neighborhoods agree with filter-neighborhoods.

Compact sets: If $X$ is net-compact, let $\mathcal{U}$ be an ultrafilter on $X$. Proposition 5.1 yields a net $\left(x_{\alpha}\right)$ in $X$ with $\mathcal{U}=\left[x_{\alpha}\right]$. Use the netcompactness of $X$ to find a quasi-subnet $\left(y_{\beta}\right)$ of $\left(x_{\alpha}\right)$ such that $y_{\beta} \rightarrow x$ for some $x$. It follows that $\mathcal{U}=\left[x_{\alpha}\right] \subseteq\left[y_{\beta}\right] \rightarrow x$. Maximality of $\mathcal{U}$ then implies $\mathcal{U}=\left[y_{\beta}\right] \rightarrow x$, so $X$ is filter-compact. Conversely, suppose that $X$ is filter-compact. Let $\left(x_{\alpha}\right)$ be a net in $X$. Then $\left[x_{\alpha}\right]$ is a filter, so it is contained in some ultrafilter $\mathcal{U}$. Then $\mathcal{U} \rightarrow x$ for some $x$. Now find a net $\left(y_{\beta}\right)$ with $\mathcal{U}=\left[y_{\beta}\right]$. We clearly have $y_{\beta} \rightarrow x$, and it follows from $\left[x_{\alpha}\right] \subseteq\left[y_{\beta}\right]$ that $\left(y_{\beta}\right)$ is a quasi-subnet of $\left(x_{\alpha}\right)$. Therefore, $X$ is net-compact.

It is straightforward to verify that the concepts of local compactness agree for nets and filters.

Bounded sets: Let $X$ be a convergence vector space and $A \subseteq X$. Suppose that $A$ is net-bounded. Since $\mathcal{N}_{0} \rightarrow 0$ in $\mathbb{K}$, we can find a net $\left(r_{\alpha}\right)$ in $\mathbb{K}$ such that $\left[r_{\alpha}\right]=\mathcal{N}_{0}$ and $r_{\alpha} \rightarrow 0$. It follows that the net $\left(r_{\alpha} a\right)_{(\alpha, a)}$ converges to zero in $X$. It is easy to verify $\mathcal{N}_{0} A=\left\langle r_{\alpha} a\right\rangle$, so $\left[\mathcal{N}_{0} A\right]=\left[r_{\alpha} a\right] \rightarrow 0$. This means that $A$ is filter-bounded. Conversely, suppose $A$ is filter-bounded and let $r_{\alpha} \rightarrow 0$ in $\mathbb{K}$. Then $\left[r_{\alpha}\right] \rightarrow 0$ means $\mathcal{N}_{0} \subseteq\left[r_{\alpha}\right]$. It follows that every $U \in \mathcal{N}_{0}$ contains a tail of $\left(r_{\alpha}\right)$ and, therefore, $U A$ contains a tail of $\left(r_{\alpha} a\right)$. This gives $\mathcal{N}_{0} A \subseteq\left\langle r_{\alpha} a\right\rangle$, so $\left[\mathcal{N}_{0} A\right] \subseteq\left[r_{\alpha} a\right]$. Now $\left[\mathcal{N}_{0} A\right] \rightarrow 0$ implies $\left[r_{\alpha} a\right] \rightarrow 0$, hence $r_{\alpha} a \rightarrow 0$, showing that $A$ is net-bounded. 
It is an easy exercise that a convergence vector space is net-locally bounded iff it is filter-locally bounded.

Mackey modification: Consider a convergence vector space $(X, \rightarrow$ ). We claim that its net-Mackey modification agrees with its filterMackey modification in the sense that $x_{\alpha} \stackrel{\mathrm{m}}{\rightarrow} x$ iff $\left[x_{\alpha}\right] \stackrel{\mathrm{m}}{\rightarrow} x$. Since both the original structure and its Mackey modification are linear, we may assume that $x=0$.

Suppose that $x_{\alpha} \stackrel{\mathrm{m}}{\rightarrow} 0$. Then there exists a bounded set $B$ such that for every $\varepsilon>0$ the set $\varepsilon B$ contains a tail of $\left(x_{\alpha}\right)$. It follows that $\varepsilon B \in\left[x_{\alpha}\right]$. Since $\varepsilon$ was arbitrary, we conclude that $\mathcal{N}_{0} B \subseteq\left[x_{\alpha}\right]$ and, therefore, $\left[x_{\alpha}\right] \stackrel{\mathrm{m}}{\rightarrow} 0$. For the converse we assume $\left[x_{\alpha}\right] \stackrel{\mathrm{m}}{\rightarrow} 0$. Find a bounded set $B$ such that $\mathcal{N}_{0} B \subseteq\left[x_{\alpha}\right]$. If $\varepsilon>0$ is arbitrary, $\varepsilon B \in \mathcal{N}_{0} B$, and hence $\varepsilon B$ contains a tail of $\left(x_{\alpha}\right)$. This yields $x_{\alpha} \stackrel{\mathrm{m}}{\rightarrow} 0$.

In view of this equivalence, Proposition 3.9 implies Proposition 3.7.17 in [BB02].

First countability. The rest of this section is devoted to translations of the first countability condition into the language of nets.

Proposition 6.1. A convergence space is first countable at $x$ iff for every net $\left(x_{\alpha}\right)_{\alpha \in \Lambda}$ which converges to $x$ there exists a net $\left(y_{\gamma}\right)_{\gamma \in \Gamma}$ such that $\left(x_{\alpha}\right) \preceq\left(y_{\gamma}\right), y_{\gamma} \rightarrow x$, and $\Gamma$ admits a countable co-final subset.

Proof. Suppose that $X$ is first countable and let $\left(x_{\alpha}\right)_{\alpha \in \Lambda} \rightarrow x$. Find a filter $\mathcal{G} \subseteq\left[x_{\alpha}\right]$ such that $\mathcal{G} \rightarrow x$ and $\mathcal{G}$ has a countable base $\left\{B_{n}\right\}$. Replacing $B_{n}$ with $B_{1} \cap \cdots \cap B_{n}$, we may assume that $\left(B_{n}\right)$ is nested and decreasing. Define $\left(y_{\gamma}\right)_{\gamma \in \Gamma}$ as in the proof of Proposition 5.1 by putting $\Gamma=\left\{(n, b): n \in \mathbb{N}, b \in B_{n}\right\}$, pre-ordering it by the first component, and letting $y_{(n, b)}=b$. Then $\left\langle y_{\gamma}\right\rangle_{\gamma \in \Gamma}=\left\{B_{n}\right\}_{n \in \mathbb{N}}$ and $\left[y_{\gamma}\right]=\mathcal{G}$. It follows that $\left(x_{\alpha}\right) \preceq\left(y_{\gamma}\right)$ and $y_{\gamma} \rightarrow x$. For every $n \in \mathbb{N}$, pick any $b_{n} \in B_{n}$; the set $\Gamma_{0}=\left\{\left(n, b_{n}\right): n \in \mathbb{N}\right\}$ is a countable co-final subset of $\Gamma$.

To prove the converse, suppose that $\mathcal{F} \rightarrow x$. Find $\left(x_{\alpha}\right)_{\alpha \in \Lambda}$ such that $\mathcal{F}=\left[x_{\alpha}\right]$. Then there exists a net $\left(y_{\gamma}\right)_{\gamma \in \Gamma}$ such that $\left(x_{\alpha}\right) \preceq\left(y_{\gamma}\right)$, $y_{\gamma} \rightarrow x$, and $\Gamma$ admits a countable co-final subset $\left\{\gamma_{n}\right\}$. Let $\mathcal{G}=\left[y_{\gamma}\right]$ and, for every $n$, put $B_{n}=\left\{y_{\gamma}\right\}_{\gamma \geqslant \gamma_{n}}$. It follows that $\mathcal{G} \rightarrow x, \mathcal{G} \subseteq \mathcal{F}$, and $\left\{B_{n}\right\}$ is a countable base of $\mathcal{G}$. 
Proposition 6.2. Let $X$ be a convergence space. If $X$ is first countable at $x$ and $\left(x_{\alpha}\right)_{\alpha \in \Lambda} \rightarrow x$ then there exists an increasing sequence of indices $\left(\alpha_{n}\right)$ in $\Lambda$ such that $x_{\alpha_{n}} \rightarrow x$. Moreover, if $\beta_{n} \geqslant \alpha_{n}$ for every $n$ then $x_{\beta_{n}} \rightarrow x$.

Proof. By first countability, there exists a filter $\mathcal{G} \subseteq\left[x_{\alpha}\right]$ such that $\mathcal{G} \rightarrow x$ and $\mathcal{G}$ has a countable base, say, $\left\{B_{n}\right\}$. Again, we may assume that $\left(B_{n}\right)$ is nested and decreasing. Inductively, we find an increasing sequence $\left(\alpha_{n}\right)$ in $\Lambda$ such that $\left\{x_{\alpha}\right\}_{\alpha \geqslant \alpha_{n}} \subseteq B_{n}$ for every $n$. It follows that $\left\{x_{\alpha_{k}}\right\}_{k \geqslant n}$ is contained in $B_{n}$ and, therefore, $B_{n}$ is in the tail filter of the sequence $\left(x_{\alpha_{n}}\right)$. This yields $\mathcal{G} \subseteq\left[x_{\alpha_{n}}\right]$, so that $\left[x_{\alpha_{n}}\right] \rightarrow x$; that is, $x_{\alpha_{n}} \rightarrow x$.

Similarly, if $\beta_{n} \geqslant \alpha_{n}$ for every $n$ then $\left\{x_{\beta_{k}}\right\}_{k \geqslant n}$ is contained in $B_{n}$, hence $\mathcal{G} \subseteq\left[x_{\beta_{n}}\right]$ and $x_{\beta_{n}} \rightarrow x$.

This immediately yields that the closure of a set in a first countable convergence space agrees with its sequential closure (Proposition 1.6.4 in [BB02]).

Definition 6.3. Let $\left(x_{\alpha}\right)_{\alpha \in \Lambda}$ be a net and $\left(\alpha_{n}\right)$ an increasing sequence of indices in $\Lambda$. Let

$$
\Gamma=\left\{(n, \alpha): n \in \mathbb{N}, \alpha \in \Lambda, \alpha \geqslant \alpha_{n}\right\}
$$

be pre-ordered by the first component. The net $y_{(n, \alpha)}=x_{\alpha}$ indexed by $\Gamma$ is called a matryoshka of $\left(x_{\alpha}\right)$.

Remark 6.4. In the preceding definition, let $\gamma_{0}=\left(n_{0}, \alpha_{0}\right) \in \Gamma$. It can be easily verified that $\left\{y_{\gamma}\right\}_{\gamma \geqslant \gamma_{0}}$ equals $\left\{x_{\alpha}\right\}_{\alpha \geqslant \alpha_{n_{0}}}$. That is, the tail sets of a matryoshka are precisely the tail sets of the original net corresponding to the $\left(\alpha_{n}\right)$.

Proposition 6.5. A convergence space is first countable at $x$ iff every net $\left(x_{\alpha}\right)$ that converges to $x$ admits a matryoshka that converges to $x$.

Proof. First we assume $X$ is first countable at $x$ and let $x_{\alpha} \rightarrow x$. Take $\mathcal{G},\left(\alpha_{n}\right)$ and $\left(B_{n}\right)$ be as in the proof of Proposition 6.2. and let $\left(y_{\gamma}\right)_{\gamma \in \Gamma}$ be the matryoshka of $\left(x_{\alpha}\right)$ corresponding to $\left(\alpha_{n}\right)$. By Remark 6.4, each $B_{n}$ contains a tail of $\left(y_{\gamma}\right)$, so $\mathcal{G} \subseteq\left[y_{\gamma}\right]$. It follows that $y_{\gamma} \rightarrow x$. 
To prove the converse, suppose that $\mathcal{F} \rightarrow x$. Find a net $\left(x_{\alpha}\right)$ such that $\mathcal{F}=\left[x_{\alpha}\right]$. By assumption, there exists an increasing sequence $\left(\alpha_{n}\right)$ in $\Lambda$ such that the corresponding matryoshka $\left(y_{\gamma}\right)_{\gamma \in \Gamma}$ converges to $x$. Then defining $\mathcal{G}=\left[y_{\gamma}\right]$ implies $\mathcal{G} \rightarrow x$. It follows from Remark 6.4 that $\mathcal{G} \subseteq \mathcal{F}$.

\section{Mixings And (pre)topological StruCtures}

In this section we present an equivalent reformulation of Axiom (N3) based on the concept of mixing. We then use this concept to characterize pretopological convergence structures in terms of nets.

Let $X$ be a set, and let $\left(x_{\alpha}\right),\left(y_{\alpha}\right)$ and $\left(z_{\alpha}\right)$ be three nets in $X$ with the same index sets. We say that $\left(z_{\alpha}\right)$ is a braiding of $\left(x_{\alpha}\right)$ and $\left(y_{\alpha}\right)$ if $z_{\alpha} \in\left\{x_{\alpha}, y_{\alpha}\right\}$ for every $\alpha$. In this terminology, Axiom (N3) effectively says that if $x_{\alpha} \rightarrow x$ and $y_{\alpha} \rightarrow x$ then every braiding of the two nets also converges to $x$.

Next, we define a closely related concept of $\boldsymbol{m i x i n g . ~ G i v e n ~ t w o ~}$ nets, $\left(x_{\alpha}\right)_{\alpha \in A}$ and $\left(y_{\beta}\right)_{\beta \in B}$, we construct a new net $\left(u_{\gamma}\right)_{\gamma \in \Gamma}$ which, in a certain sense, combines $\left(x_{\alpha}\right)$ and $\left(y_{\beta}\right)$ in a single net. Set

$$
\Gamma=\left\{(\alpha, \beta, z): \alpha \in A, \beta \in B, z \in\left\{x_{\alpha}, y_{\beta}\right\}\right\}
$$

and give it the following pre-order: $\left(\alpha_{1}, \beta_{1}, z_{1}\right) \leqslant\left(\alpha_{2}, \beta_{2}, z_{2}\right)$ whenever $\alpha_{1} \leqslant \alpha_{2}$ and $\beta_{1} \leqslant \beta_{2}$. It is clear that $\Gamma$ is a directed set. We now put $u_{(\alpha, \beta, z)}=z$. The resulting net is called the mixing of $\left(x_{\alpha}\right)$ and $\left(y_{\beta}\right)$.

The following two lemmas characterize the relationships between braiding and mixing.

Lemma 7.1. Every braiding of $\left(x_{\alpha}\right)_{\alpha \in A}$ and $\left(y_{\alpha}\right)_{\alpha \in A}$ is a quasi-subnet of their mixing.

Proof. Let $\left(z_{\alpha}\right)_{\alpha \in A}$ be a braiding of $\left(x_{\alpha}\right)_{\alpha \in A}$ and $\left(y_{\alpha}\right)_{\alpha \in A}$, and let $\left(u_{\gamma}\right)_{\gamma \in \Gamma}$ be the mixing of $\left(x_{\alpha}\right)_{\alpha \in A}$ and $\left(y_{\alpha}\right)_{\alpha \in A}$. For every $\alpha \in A$, the triple $\gamma=\left(\alpha, \alpha, z_{\alpha}\right)$ belongs to $\Gamma$ and $u_{\gamma}=z_{\alpha}$. Put

$$
\Gamma_{0}=\left\{\left(\alpha, \alpha, z_{\alpha}\right): \alpha \in A\right\} .
$$

It is easy to see that $\Gamma_{0}$ is a directed subset of $\Gamma$. Moreover, $\Gamma_{0}$ is

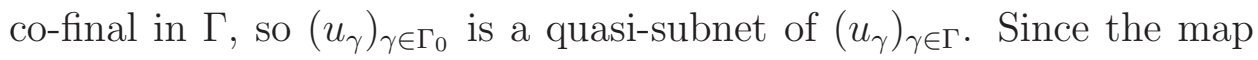


$\alpha \mapsto\left(\alpha, \alpha, z_{\alpha}\right)$ is an order isomorphism between $A$ and $\Gamma_{0}$, the nets $\left(z_{\alpha}\right)_{\alpha \in A}$ and $\left(u_{\gamma}\right)_{\gamma \in \Gamma_{0}}$ are the same net up to this isomorphism between the index sets.

Lemma 7.2. The mixing of two nets is a braiding of a pair of nets that are strongly tail equivalent to the original ones.

Proof. Let $\left(u_{\gamma}\right)_{\gamma \in \Gamma}$ be the mixing of $\left(x_{\alpha}\right)_{\alpha \in A}$ and $\left(y_{\beta}\right)_{\beta \in B}$. Define two nets $\left(x_{\gamma}^{\prime}\right)_{\gamma \in \Gamma}$ and $\left(y_{\gamma}^{\prime}\right)_{\gamma \in \Gamma}$ as follows: for $\gamma=(\alpha, \beta, z) \in \Gamma$, we put $x_{\gamma}^{\prime}=x_{\alpha}$ and $y_{\gamma}^{\prime}=y_{\beta}$. By the definition of $\Gamma$, we have

$$
u_{\gamma}=z \in\left\{x_{\alpha}, y_{\beta}\right\}=\left\{x_{\gamma}^{\prime}, y_{\gamma}^{\prime}\right\}
$$

hence $\left(u_{\gamma}\right)$ is a braiding of $\left(x_{\gamma}^{\prime}\right)$ and $\left(y_{\gamma}^{\prime}\right)$. On the other hand, it is easy to see that $\left(x_{\gamma}^{\prime}\right)_{\gamma \in \Gamma}$ and $\left(y_{\gamma}^{\prime}\right)_{\gamma \in \Gamma}$ are strongly tail equivalent to $\left(x_{\alpha}\right)_{\alpha \in A}$ and $\left(y_{\alpha}\right)_{\beta \in B}$, respectively.

Combining the two lemmas, we immediately get the following.

Theorem 7.3. Axiom (N3) in the definition of net convergent structures may be replaced with

$\left(\mathrm{N} 3^{*}\right)$ If two nets converge to $x$ then their mixing converges to $x$.

We now extend the definition of mixing to an arbitrary set of nets. For these purposes, it is useful to introduce some notation.

Definition 7.4. Let $\Sigma=\left\{S_{j}: j \in J\right\}$ be a set of nets indexed by a set $J$; so $S_{j}$ denotes a net for each $j \in J$. Note that we do not assume any structure on $J$. Each net in $\Sigma$ is associated with a directed pre-ordered index set $A_{j}$; i.e., $S_{j}=\left(x_{\alpha^{(j)}}\right)_{\alpha^{(j)} \in A_{j}}$ for each $j \in J$. Define $\Gamma=\left(\prod_{j \in J} A_{j}\right) \times J$ and equip it with the product pre-order on the first component: for $\gamma_{1}=\left(\bar{\alpha}_{1}, j_{1}\right)$ and $\gamma_{2}=\left(\bar{\alpha}_{2}, j_{2}\right)$, we have $\gamma_{1} \leqslant \gamma_{2}$ whenever $\alpha_{1}^{(j)} \leqslant \alpha_{2}^{(j)}$ for all $j \in J$. It is easy to see that $\Gamma$ is directed. Now put $\gamma=(\bar{\alpha}, j)$ and define $y_{\gamma}=x_{\bar{\alpha}(j)}$; this net $M=\left(y_{\gamma}\right)_{\gamma \in \Gamma}$ is called the mixing of $\Sigma$. When we do not wish to draw attention to the index sets of the nets in $\Sigma$, we will suppress the reference to the index set $J$ and just write $S \in \Sigma$ to denote a net from the family.

It is easy to see this mixing net $M$ visits every term of every net $S$ in $\Sigma$. Moreover, it visits them in the same order. Also note the 
definition of mixing for arbitrary sets of nets agrees with the mixing of two nets from before.

Lemma 7.5. Let $\Sigma$ be a family of nets in $X$ and $M$ the mixing of $\Sigma$. Then each of the nets that make up $\Sigma$ is a quasi-subnet of $M$.

Proof. Using the notation above, fix $j_{0} \in J$ and let $\Gamma_{0} \subseteq \Gamma$ be the " $j_{0}$-th column" of $\Gamma: \Gamma_{0}=\left(\prod_{j \in J} A_{j}\right) \times\left\{j_{0}\right\}$. It is easy to see that $\Gamma_{0}$ is a directed co-final subset of $\Gamma$, hence $\left(y_{\gamma}\right)_{\gamma \in \Gamma_{0}}$ is a quasi-subnet of $M$. On the other hand, $\left(y_{\gamma}\right)_{\gamma \in \Gamma_{0}}$ has the same terms as $S_{j_{0}}$ and the two nets are strongly tail equivalent.

Lemma 7.6. Let $\Sigma$ be a family of nets in $X$ and $M$ the mixing of $\Sigma$. Then the tail filter of $M$ is the intersection of the tail filters of the nets that make up $\Sigma$.

Proof. Recall that we write $[M]$ for the tail filter of $M$. Thus, we need to show that $[M]=\bigcap_{S \in \Sigma}[S]$.

Let $A \in[M]$. Then $A$ contains a tail of $M$, hence a tail of every $S \in \Sigma$, because each $S$ is a quasi-subnet of $M$ by the Lemma above.

Conversely, suppose $A \in \bigcap_{S \in \Sigma}[S]$. For every $S \in \Sigma, A$ contains a tail of $S$. It is a routine verification that the mixing of these tails is a tail of $M$ that is still contained in $A$.

Recall that if $X$ is a (filter) convergence space and $x \in X$, the neighborhood filter $\mathcal{U}_{x}$ of $x$ is defined as the intersection of all filters that converge to $x$. A convergence structure is pretopological if $\mathcal{U}_{x} \rightarrow x$ for every $x$. This terminology is motivated by the situation in topological convergence structures: the definition of $\mathcal{U}_{x}$ agrees with the usual neighborhood filter $\mathcal{N}_{x}$, which converges to $x$. We are now ready to show that extending $\left(\mathrm{N}^{*}\right)$ from two nets to an arbitrary collection of nets characterizes pretopological convergence structures in terms of nets.

Theorem 7.7. A convergence space $X$ is pretopological iff for every $x \in X$ and every family of nets converging to $x$, the mixing of the family also converges to $x$. 
Proof. Suppose that the convergence structure is pretopological. Let $\Sigma$ be a collection of nets converging to $x$ where $M$ is the mixing of $\Sigma$. For every $S \in \Sigma$ we have $S \rightarrow x$, hence $[S] \rightarrow x$. It follows from $[M]=\bigcap_{S \in \Sigma}[S] \supseteq \mathcal{U}_{x} \rightarrow x$ that $[M] \rightarrow x$ and, therefore, $M \rightarrow x$.

To prove the converse, note that for each filter $\mathcal{F}$ that converges to $x$ we can find a net $S$ such that $S \rightarrow x$ and $\mathcal{F}=[S]$. Denote the resulting collection of nets by $\Sigma$ and let $M$ be the mixing of $\Sigma$. Then $M \rightarrow x$ and we get $\mathcal{U}_{x}=\bigcap_{S \in \Sigma}[S]=[M] \rightarrow x$.

We now characterize topological convergence structures in similar terms. There is extensive literature on this subject: [KN76, p. 74], [AA72], Sch97, 15.10], and [BB02] are some examples. Of note, Proposition 1.3.21 of [BB02] shows that a convergence structure is topological iff it is pretopological and the closure of every set is closed. Compare this to [KN76, p. 69], which proves that a convergence structure is topological when it satisfies a certain iterated limit property. In the case of sequences, this essentially means the limit of a double sequence $\left(x_{n, m}\right)$ agrees with its iterated $\operatorname{limit}$; i.e., $\lim x_{m, n}=\lim _{m} \lim x_{m, n}$ whenever the latter limit exists. For nets, the iterated limit property is closely related to the concept of mixing.

As in Definition 7.4, we let $\Sigma=\left\{S_{j}: j \in J\right\}$ denote a set of nets and emphasize the index sets $A_{j}$ for each net $S_{j}$; that is, $S_{j}=\left(x_{\alpha^{(j)}}\right)_{\alpha^{(j)} \in A_{j}}$ for each $j \in J$. The only difference now is that we make the additional assumption that $J$ is a directed pre-ordered set. This allows us to view $\Sigma$ as a net $\left(S_{j}\right)_{j \in J}$ of nets. Take $\Delta=\left(\prod_{j \in J} A_{j}\right) \times J$ and equip it with the product pre-order: for $\gamma_{1}=\left(\bar{\alpha}_{1}, j_{1}\right)$ and $\gamma_{2}=\left(\bar{\alpha}_{2}, j_{2}\right)$ we declare $\gamma_{1} \leqslant \gamma_{2}$ whenever $\alpha_{1}^{(j)} \leqslant \alpha_{2}^{(j)}$ for all $j \in J$ and $j_{1} \leqslant j_{2}$. It is easy to see that this makes $\Delta$ into a directed set. For $\rho=(\bar{\alpha}, j) \in \Delta$, put $r_{\rho}=x_{\bar{\alpha}(j)}$. The resulting net $R=\left(r_{\rho}\right)_{\rho \in \Delta}$ is called the reaction of $J$ with $\Sigma$.

Note that as a set, $\Delta$ coincides with $\Gamma$ in the definition of mixing; however, the two differ as directed sets: the only difference is $\Delta$ can "see" an order on $J$. Similarly, $R$ and the mixing of $\Sigma$ agree as functions but are distinct as nets: $R$ is a quasi-subnet of $M$. 
We say that a convergence structure has the iterated limit property if $\lim R=\lim _{j \in J} \lim S_{j}$, whenever the limit in the right hand side exists, for every net $\Sigma$ of nets in $X$.

The following result is essentially contained in [Sch97, 15.10], but we present a shorter proof.

Theorem 7.8. A convergence structure $X$ is topological iff it satisfies the iterated limit property.

Proof. The forward implication is proved in [KN76, p. 69]. For the converse, suppose that $X$ has the iterated limit property. It suffices to verify that $X$ is pretopological and the closure of every set is closed. The latter condition is straightforward. To prove that $X$ is pretopological we use Theorem [7.7, Let $\Sigma=\left\{S_{j}: j \in J\right\}$ be as in Definition 7.4, and let $M$ be the mixing of $\Sigma$. Assume that $S_{j} \rightarrow x$ for every $j \in J$. We will show $M \rightarrow x$. Give $J$ the trivial pre-order by declaring $j_{1} \leqslant j_{2}$ for every $j_{1}, j_{2} \in J$, and let $R$ be the reaction of $J$ with $\Sigma$. Then $R \rightarrow x$ by the iterated limit property. However, it is easy to see that under our pre-ordering of $J, M$ and $R$ agree as nets. It immediately follows that $M \rightarrow x$.

\section{Continuous Convergence}

In this section we apply net convergence theory to reprove some results about continuous convergence from [BB02]. While the results themselves are nothing new, our arguments highlight the simplicity of the net language for functional analysis.

Let $X$ and $Y$ be two convergence spaces, and use $C(X, Y)$ to denote the set of all continuous functions from $X$ to $Y$. Continuous convergence on $C(X, Y)$ is defined as follows: $f_{\alpha} \stackrel{\text { c }}{\rightarrow} f$ if $f_{\alpha}\left(x_{\beta}\right) \rightarrow f(x)$ in $Y$ whenever $x_{\beta} \rightarrow x$ in $X$. Note that we treat $\left(f_{\alpha}\left(x_{\beta}\right)\right)_{(\alpha, \beta)}$ as a net over the product of the index sets. Note also that this net need not be admissible. It is easy to verify that continuous convergence is a net convergence structure. One should compare this with the definition in terms of filters: for a filter $\Phi$ on $C(X, Y), \Phi \stackrel{\text { c }}{\rightarrow} f$ if $\Phi(\mathcal{F}) \rightarrow f(x)$ whenever $\mathcal{F} \rightarrow x$; here $\mathcal{F}$ is a filter on $X$ and $\Phi(\mathcal{F})$ is the filter generated by the sets of the form $F(A)$ where $F \in \Phi$ and $A \in \mathcal{F}$. 
These definitions are equivalent in the sense that $\Phi \stackrel{\text { c }}{\rightarrow} f$ iff $\Phi=\left[f_{\alpha}\right]$ for some net $\left(f_{\alpha}\right)$ with $f_{\alpha} \stackrel{\text { c }}{\rightarrow} f$. This is an easy corollary of the fact that $\Phi(\mathcal{F})=\left[f_{\alpha}\left(x_{\beta}\right)\right]$ whenever $\Phi=\left[f_{\alpha}\right]$ and $\mathcal{F}=\left[x_{\beta}\right]$, where $\left(f_{\alpha}\right)$ is a net in $C(X, Y)$ and $\left(x_{\beta}\right)$ a net in $X$.

We will write $C_{c}(X, Y)$ for the set $C(X, Y)$ equipped with the continuous convergence structure. We will write $C_{c}(X)$ in place of $C_{c}(X, \mathbb{K})$ where the scalar field is always assumed to carry the standard convergence. It is easy to see that $C_{c}(X)$ is a convergence vector space.

The next few results remain valid, with similar proofs, if we replace $C_{c}(X)$ with $C_{c}(X, Y)$ where $Y$ is a Hausdorff complete topological vector space. We use $C_{c}(X)$ rather than $C_{c}(X, Y)$ for simplicity.

Proposition 8.1. For every convergence space $X, C_{c}(X)$ is complete.

Proof. The proof is a standard approximation argument. Let $\left(f_{\alpha}\right)_{\alpha \in \Lambda}$ be Cauchy net in $C_{c}(X)$. In particular, for every $x \in X$, the double net $\left(f_{\alpha}(x)-f_{\beta}(x)\right)_{(\alpha, \beta)}$ converges to zero in $\mathbb{K}$. It follows that the net $\left(f_{\alpha}(x)\right)$ is Cauchy and, therefore, converges to a unique limit in the usual convergence of $\mathbb{K}$. Write $f(x)=\lim _{\alpha} f_{\alpha}(x)$. It is left to show that $f$ is continuous and $f_{\alpha} \stackrel{\mathrm{c}}{\rightarrow} f$.

Let $\left(x_{\gamma}\right)_{\gamma \in \Gamma}$ be a net in $X$ with $x_{\gamma} \rightarrow x$. It follows from $f_{\alpha}-f_{\beta} \stackrel{\text { c }}{\rightarrow} 0$ that $f_{\alpha}\left(x_{\gamma}\right)-f_{\beta}\left(x_{\gamma}\right) \rightarrow 0$ in $\mathbb{K}$, where the expression is a net over $\Lambda \times \Lambda \times \Gamma$. Fix $\varepsilon>0$. There exist $\alpha_{0}, \beta_{0}$, and $\gamma_{0}$ such that

$$
\left|f_{\alpha}\left(x_{\gamma}\right)-f_{\beta}\left(x_{\gamma}\right)\right|<\varepsilon
$$

whenever $\alpha \geqslant \alpha_{0}, \beta \geqslant \beta_{0}$, and $\gamma \geqslant \gamma_{0}$.

Fix $\beta_{1} \geqslant \beta_{0}$ such that

$$
\left|f_{\beta_{1}}(x)-f(x)\right|<\varepsilon ;
$$

this can be done by the definition of $f(x)$. Since $f_{\beta_{1}}$ is continuous, we can find $\gamma_{1} \geqslant \gamma_{0}$ such that

$$
\left|f_{\beta_{1}}\left(x_{\gamma}\right)-f_{\beta_{1}}(x)\right|<\varepsilon
$$

whenever $\gamma \geqslant \gamma_{1}$. Fix $\gamma \geqslant \gamma_{1}$. Using the definition of $f$ again, we find some $\alpha_{1} \geqslant \alpha_{0}$ such that

$$
\left|f_{\alpha_{1}}\left(x_{\gamma}\right)-f\left(x_{\gamma}\right)\right|<\varepsilon
$$


Combining these inequalities, we get

$$
\begin{aligned}
\left|f\left(x_{\gamma}\right)-f(x)\right| \leqslant\left|f\left(x_{\gamma}\right)-f_{\alpha_{1}}\left(x_{\gamma}\right)\right|+\left|f_{\alpha_{1}}\left(x_{\gamma}\right)-f_{\beta_{1}}\left(x_{\gamma}\right)\right| \\
+\left|f_{\beta_{1}}\left(x_{\gamma}\right)-f_{\beta_{1}}(x)\right|+\left|f_{\beta_{1}}(x)-f(x)\right|<4 \varepsilon .
\end{aligned}
$$

It follows that $f\left(x_{\gamma}\right) \rightarrow f(x)$, hence $f$ is continuous. Furthermore, it follows from the first three inequalities that for every $\alpha \geqslant \alpha_{0}$ and every $\gamma \geqslant \gamma_{1}$ we have

$\left|f_{\alpha}\left(x_{\gamma}\right)-f(x)\right| \leqslant\left|f_{\alpha}\left(x_{\gamma}\right)-f_{\beta_{1}}\left(x_{\gamma}\right)\right|+\left|f_{\beta_{1}}\left(x_{\gamma}\right)-f_{\beta_{1}}(x)\right|+\left|f_{\beta_{1}}(x)-f(x)\right|<3 \varepsilon$,

which yields $f_{\alpha} \stackrel{\mathrm{c}}{\rightarrow} f$.

We now define uniform convergence on compacta (ucc). Let $X$ be a convergence space and $f \in C(X)$. For a net $\left(f_{\alpha}\right)$ in $C(X)$, we write $f_{\alpha} \stackrel{\text { ucc }}{\longrightarrow} f$ if $f_{\alpha}$ converges to $f$ uniformly on every compact subset of $X$. Note that ucc convergence on $C(X)$ is topological and locally convex; this topology has a base of zero neighborhoods given by the sets

$$
V_{A, \varepsilon}=\{f \in C(X):|f(x)|<\varepsilon \text { for all } x \in A\},
$$

where $\varepsilon$ is a positive real and $A$ is a compact subset of $X$.

Proposition 8.2. Let $X$ be a convergence space. If $f_{\alpha} \stackrel{\mathrm{c}}{\rightarrow} f$ then $f_{\alpha} \stackrel{\text { ucc }}{\longrightarrow} f$ in $C(X)$.

Proof. By linearity we may assume $f=0$. For the sake of contradiction, let $\left(f_{\alpha}\right)_{\alpha \in \Lambda}$ be a net in $C(X)$ such that $f_{\alpha} \stackrel{\mathrm{c}}{\rightarrow} 0$ but $f_{\alpha}$ fails to converge to 0 uniformly on some compact subset $A$ of $X$. It follows that there exists $\varepsilon>0$ such that for every $\alpha \in \Lambda$ there exists $x_{\alpha} \in A$ and $\beta=\beta(\alpha)$ in $\Lambda$ with $\beta(\alpha) \geqslant \alpha$ such that $\left|f_{\beta(\alpha)}\left(x_{\alpha}\right)\right|>\varepsilon$. Then $g_{\alpha}=f_{\beta(\alpha)}$ defines a quasi-subnet of $\left(f_{\alpha}\right)$, hence $g_{\alpha} \stackrel{\mathrm{c}}{\rightarrow} 0$. Employing the compactness of $A$ and passing to a further quasi-subnet, we may assume $\left(x_{\alpha}\right)$ is convergent. From this we obtain $g_{\alpha}\left(x_{\alpha}\right) \rightarrow 0$, which contradicts $\left|g_{\alpha}\left(x_{\alpha}\right)\right|=\left|f_{\beta(\alpha)}\left(x_{\alpha}\right)\right|>\varepsilon$ for all $\alpha$.

Corollary 8.3. Let $X$ be a locally compact convergence space. Then the continuous and the ucc convergence structures on $C(X)$ agree. In particular, $C_{c}(X)$ is a locally convex topological vector space. 
Proof. We already know that $f_{\alpha} \stackrel{\mathrm{c}}{\rightarrow} f$ implies $f_{\alpha} \stackrel{\text { ucc }}{\longrightarrow} f$. Suppose that $f_{\alpha} \stackrel{\text { ucc }}{\longrightarrow} f$ in $C_{c}(X)$ and $x_{\beta} \rightarrow x$ in $X$. Fix $\varepsilon>0$. Since $X$ is locally compact, there exists a compact set $A$ in $X$ and an index $\beta_{0}$ such that $x_{\beta} \in A$ for all $\beta \geqslant \beta_{0}$. The continuity of $f$ yields some $\beta_{1}$ such that $\left|f\left(x_{\beta}\right)-f(x)\right|<\varepsilon$ whenever $\beta \geqslant \beta_{1}$. Since $f_{\alpha} \stackrel{\text { ucc }}{\longrightarrow} f$, there exists an index $\alpha_{0}$ such that $\left|f_{\alpha}(z)-f(z)\right|<\varepsilon$ for all $\alpha \geqslant \alpha_{0}$ and all $z \in A$. It follows that for all $\alpha \geqslant \alpha_{0}$ and all $\beta$ such that $\beta \geqslant \beta_{0}$ and $\beta \geqslant \beta_{1}$, we have

$$
\left|f_{\alpha}\left(x_{\beta}\right)-f(x)\right| \leqslant\left|f_{\alpha}\left(x_{\beta}\right)-f\left(x_{\beta}\right)\right|+\left|f\left(x_{\beta}\right)-f(x)\right|<2 \varepsilon
$$

implying $f_{\alpha}\left(x_{\beta}\right) \rightarrow f(x)$.

Let $X$ be a convergence vector space. Following [BB02], we write $\mathcal{L}_{c} X$ for the space of all continuous linear functionals on $X$ equipped with the continuous convergence structure. Note that $\mathcal{L}_{c} X$ is a closed subspace of $C_{c}(X)$. Then Proposition 8.1 implies $\mathcal{L}_{c} X$ is complete. One may view $\mathcal{L}_{c} X$ as the dual space of $X$ in the category of convergence vector spaces.

Let $X$ be a topological vector space. As a set, $\mathcal{L}_{c} X$ agrees with the topological dual $X^{*}$ of $X$. The next proposition compares the continuous convergence on $\mathcal{L}_{c} X$ with the weak ${ }^{*}$-convergence, i.e., convergence in the $\sigma\left(X^{*}, X\right)$-topology.

Proposition 8.4. Let $X$ be a topological vector space. Then $f_{\alpha} \stackrel{\text { c }}{\rightarrow} f$ in $X^{*}$ iff $f_{\alpha} \stackrel{\mathrm{w}^{*}}{\longrightarrow} f$ and a tail of $\left(f_{\alpha}\right)$ is contained in the polar of a zero neighborhood in $X$.

Proof. Suppose $f_{\alpha} \stackrel{\mathrm{c}}{\rightarrow} f$. It is immediate that $f_{\alpha} \stackrel{\mathrm{w}^{*}}{\rightarrow} f$. Let $\mathcal{N}_{0}^{X}$ be the filter of all zero neighborhoods in $X$. Since $\mathcal{N}_{0}^{X} \rightarrow 0$, we can find a net $\left(x_{\beta}\right)$ in $X$ such that $x_{\beta} \rightarrow 0$ and $\left[x_{\beta}\right]=\mathcal{N}_{0}^{X}$. It follows that $f_{\alpha}\left(x_{\beta}\right) \rightarrow 0$. Then there exists $\alpha_{0}$ and $\beta_{0}$ such that $\left|f_{\alpha}\left(x_{\beta}\right)\right| \leqslant 1$ whenever $\alpha \geqslant \alpha_{0}$ and $\beta \geqslant \beta_{0}$. Set $V=\left\{x_{\beta}\right\}_{\beta \geqslant \beta_{0}}$ and observe $V \in \mathcal{N}_{0}^{X}$ and $\left\{f_{\alpha}\right\}_{\alpha \geqslant \alpha_{0}} \subseteq V^{\circ}$.

Conversely, suppose that $f_{\alpha} \stackrel{\mathrm{w}^{*}}{\longrightarrow} f$ and a tail $\left\{f_{\alpha}\right\}_{\alpha \geqslant \alpha_{0}}$ is contained in $V^{\circ}$ for some $V \in \mathcal{N}_{0}^{X}$. Suppose $x_{\beta} \rightarrow x$, and let $\varepsilon>0$ be fixed. Find $\alpha_{1} \geqslant \alpha_{0}$ such that $\left|f_{\alpha}(x)-f(x)\right|<\varepsilon$ whenever $\alpha \geqslant \alpha_{1}$, and find $\beta_{1}$ 
such that $x_{\beta}-x \in \varepsilon V$ for all $\beta \geqslant \beta_{1}$. Now $\alpha \geqslant \alpha_{1}$ and $\beta \geqslant \beta_{1}$ imply

$$
\left|f_{\alpha}\left(x_{\beta}\right)-f(x)\right| \leqslant\left|f_{\alpha}\left(x_{\beta}-x\right)\right|+\left|f_{\alpha}(x)-f(x)\right|<2 \varepsilon .
$$

Hence, $f_{\alpha}\left(x_{\beta}\right) \rightarrow f(x)$.

Proposition 8.5. Let $X$ be a topological vector space. The polar of every zero neighborhood in $X$ is compact in $\mathcal{L}_{c} X$. Consequently, $\mathcal{L}_{c} X$ is locally compact.

Proof. Let $V \in \mathcal{N}_{0}^{X}$. That $V^{\circ}$ is $\mathrm{w}^{*}$-compact is standard; see, e.g., page 139 in [KN76]. Indeed, $V^{\circ}$ is $\mathrm{w}^{*}$-bounded and, since the $\mathrm{w}^{*}$ topology on $X^{*}$ is the restriction to $X^{*}$ of the product topology on $\mathbb{K}^{X}$, it is relatively $\mathrm{w}^{*}$-compact. Now $V^{\circ}$ is $\mathrm{w}^{*}$-closed implies it is $\mathrm{w}^{*}$-compact. It follows that every net $\left(f_{\alpha}\right)$ in $V^{\circ}$ has a $\mathrm{w}^{*}$-convergent quasi-subnet $g_{\beta} \stackrel{\mathrm{w}^{*}}{\rightarrow} g$ for some $g \in V^{\circ}$. Proposition 8.4 gives $g_{\beta} \stackrel{\mathrm{c}}{\rightarrow} g$, implying $V^{\circ}$ is compact in $\mathcal{L}_{c} X$.

We have just shown that every continuously convergent net in $\mathcal{L}_{c} X$ has a tail inside a continuously compact set. An application of Proposition 8.4 gives $\mathcal{L}_{c} X$ is locally compact.

Corollary 8.6. If $X$ is a topological vector space then $\mathcal{L}_{c} \mathcal{L}_{c} X$ is a locally convex topological vector space with the topology of uniform convergence on compact sets in $\mathcal{L}_{c} X$.

Proof. By Proposition $8.5, \mathcal{L}_{c} X$ is locally compact. Corollary 8.3 implies that $C_{c}\left(\mathcal{L}_{c} X\right)$ is a locally convex topological vector space with the topology of uniform convergence on compact sets in $\mathcal{L}_{c} X$. The conclusion now follows from the fact that $\mathcal{L}_{c} \mathcal{L}_{c} X$ is a subspace of $C_{c}\left(\mathcal{L}_{c} X\right)$.

Proposition 8.7. Let $X$ be a topological vector space and $\left(\varphi_{\alpha}\right)$ a net in $\mathcal{L}_{c} \mathcal{L}_{c} X$. Then $\varphi_{\alpha} \stackrel{\mathrm{c}}{\rightarrow} 0$ iff $\left(\varphi_{\alpha}\right)$ converges to zero uniformly on $V^{\circ}$ for every zero neighborhood $V$ in $X$.

Proof. If $\varphi_{\alpha} \stackrel{\text { c }}{\rightarrow} 0$ then it converges to zero uniformly on compact sets by Corollary 8.3 , and $V^{\circ}$ is compact by Proposition 8.5 . To prove the converse, suppose that $\left(\varphi_{\alpha}\right)$ converges to zero uniformly on polars of zero neighborhoods in $X$. Let $\left(f_{\beta}\right)$ be a continuously convergent net in 
$\mathcal{L}_{c} X$, and let $\varepsilon>0$ be arbitrary. Proposition 8.4 guarantees that there exists a zero neighborhood $V$ in $X$ and an index $\beta_{0}$ such that $f_{\beta} \in V^{\circ}$ for all $\beta \geqslant \beta_{0}$. Since $\left(\varphi_{\alpha}\right)$ converges to zero uniformly on $V^{\circ}$, we can find $\alpha_{0}$ such that $\left|\varphi_{\alpha}(f)\right|<\varepsilon$ for all $\alpha \geqslant \alpha_{0}$ and $f \in V^{\circ}$. It follows that $\left|\varphi_{\alpha}\left(f_{\beta}\right)\right|<\varepsilon$ for all $\alpha \geqslant \alpha_{0}$ and $\beta \geqslant \beta_{0}$.

Let $X$ be a convergence vector space. For every $x \in X$, the evaluation map $\hat{x}: \mathcal{L}_{c} X \rightarrow \mathbb{K}$ given by $\hat{x}(f)=f(x)$ is continuous, hence $\hat{x} \in \mathcal{L}_{c} \mathcal{L}_{c} X$. This map is clearly linear. It follows easily from the definition of continuous convergence that the map $j: X \rightarrow \mathcal{L}_{c} \mathcal{L}_{c} X$ given by $j(x)=\hat{x}$ is continuous. We say that $X$ is reflexive if $j$ is an isomorphism. Since $\mathcal{L}_{c} X$ is complete for every convergence vector space $X$, we immediately get the following:

Proposition 8.8. Every reflexive convergence vector space is complete.

Proposition 8.9. Let $X$ be a convergence vector space. The map $f \mapsto \hat{f}$ is an isomorphic embedding of $\mathcal{L}_{c} X$ into $\mathcal{L}_{c} \mathcal{L}_{c} \mathcal{L}_{c} X$.

Proof. We already know that this map is linear and continuous. It is one-to-one: if $\hat{f}=0$ then $0=\hat{f}(\hat{x})=f(x)$ for every $x \in X$, so that $f=0$. Finally, suppose that $\hat{f}_{\alpha} \stackrel{\mathrm{c}}{\rightarrow} 0$ in $\mathcal{L}_{c} \mathcal{L}_{c} \mathcal{L}_{c} X$. Let $x_{\beta} \rightarrow 0$ in $X$. Then $\hat{x}_{\beta} \stackrel{\mathrm{c}}{\rightarrow} 0$ in $\mathcal{L}_{c} \mathcal{L}_{c} X$, so that $f_{\alpha}\left(x_{\beta}\right)=\hat{f}_{\alpha}\left(\hat{x}_{\beta}\right) \rightarrow 0$. Therefore, $f_{\alpha} \stackrel{\mathrm{c}}{\rightarrow} 0$ in $\mathcal{L}_{c} X$.

For every convergence vector space $X$, one may view $\left\langle\mathcal{L}_{c} X, \mathcal{L}_{c} \mathcal{L}_{c} X\right\rangle$ as a dual pair of vector spaces for every convergence vector space $X$ in the sense of pp. 144-145 in AB06. We will make use of the classical Mackey-Arens Theorem (see Theorem 3.24 on p. 150 of [AB06]).

Theorem 8.10 (Mackey-Arens). Let $\langle X, Y\rangle$ be a dual pair of vector spaces and $\tau$ a locally convex topology on $X$. Then the dual of $(X, \tau)$ is $Y$ iff $\tau$ is the topology of uniform convergence on some collection of convex circled $\sigma(Y, X)$-compacts subsets of $Y$.

Theorem 8.11. If $X$ is a topological vector space then $\mathcal{L}_{c} X$ is reflexive.

Proof. By Proposition 8.9 it suffices to show that the map $f \mapsto \hat{f}$ is onto. Corollary 8.6 implies $\mathcal{L}_{c} \mathcal{L}_{c} X$ is a locally convex topological vector space; denote its topology by $\tau^{* *}$. Consider the dual pair 
$\left\langle\mathcal{L}_{c} \mathcal{L}_{c} X, \mathcal{L}_{c} X\right\rangle$. If $f_{\alpha} \stackrel{\mathrm{c}}{\rightarrow} 0$ in $\mathcal{L}_{c} X$ then $\varphi\left(f_{\alpha}\right) \rightarrow 0$ for every $\varphi \in \mathcal{L}_{c} \mathcal{L}_{c} X$, hence, $\left(f_{\alpha}\right)$ converges to zero in $\sigma\left(\mathcal{L}_{c} X, \mathcal{L}_{c} \mathcal{L}_{c} X\right)$. It follows that the identity map on $\mathcal{L}_{c} X$ is continuous as a map from the continuous convergence structure to $\sigma\left(\mathcal{L}_{c} X, \mathcal{L}_{c} \mathcal{L}_{c} X\right)$.

Let $V$ be a zero neighborhood in $X$. Then $V^{\circ}$ is convex and circled. Furthermore, $V^{\circ}$ is compact in $\mathcal{L}_{c} X$ by Proposition 8.5. By the preceding paragraph, $V^{\circ}$ is $\sigma\left(\mathcal{L}_{c} X, \mathcal{L}_{c} \mathcal{L}_{c} X\right)$-compact. Let $\mathcal{S}$ be the set of all polars of zero neighborhoods in $X$. Then $\mathcal{S}$ is a set of convex, circled, and $\sigma\left(\mathcal{L}_{c} X, \mathcal{L}_{c} \mathcal{L}_{c} X\right)$-compact subsets of $\mathcal{L}_{c} X$. By Proposition 8.7, $\tau^{* *}$ is the topology of uniform convergence on sets in $\mathcal{S}$. By the MackeyArens Theorem, the dual of $\left(\mathcal{L}_{c} \mathcal{L}_{c} X, \tau^{* *}\right)$ is $\mathcal{L}_{c} X$.

Since the convergence on $\mathcal{L}_{c} \mathcal{L}_{c} X$ is topological, its continuous and topological dual agree as sets. It follows that $\mathcal{L}_{c} \mathcal{L}_{c} \mathcal{L}_{c} X$ and $\mathcal{L}_{c} X$ agree as sets. This means that the map $f \mapsto \hat{f}$ is onto.

Theorem 8.12. Let $(X, \tau)$ be a locally convex topological vector space. Then $\mathcal{L}_{c} \mathcal{L}_{c} X$ is the completion of $X$. In particular, $X$ is reflexive iff it is complete.

Proof. Let $j: X \rightarrow \mathcal{L}_{c} \mathcal{L}_{c} X$ be as before. Recall that $\mathcal{L}_{c} X$ and $X^{*}$ agree as sets. By Corollary 8.6, $\mathcal{L}_{c} \mathcal{L}_{c} X$ is a locally convex topological vector space whose topology will be denoted by $\tau^{* *}$. We already know that $j$ is continuous as a map from $(X, \tau)$ to $\left(\mathcal{L}_{c} \mathcal{L}_{c} X, \tau^{* *}\right)$. It is one-to-one: if $\hat{x}=0$ then $f(x)=\hat{x}(f)=0$ for all $f \in X^{*}$, so that $x=0$.

Next, we claim that $j$ is a homeomorphism. Suppose $\hat{x}_{\alpha} \stackrel{\text { c }}{\rightarrow} 0$ in $\mathcal{L}_{c} \mathcal{L}_{c} X$, but $x_{\alpha} \nrightarrow \rightarrow 0$ in $X$. Passing to a quasi-subnet, we find a convex circled neighborhood $V$ of zero in $X$ such that $x_{\alpha} \notin V$ for all $\alpha$. By Hahn-Banach Theorem, for every $\alpha$, we can find $f_{\alpha} \in X^{*}$ such that $f_{\alpha}\left(x_{\alpha}\right) \geqslant 1$ but $\left|f_{\alpha}(x)\right| \leqslant 1$ for all $x \in V$, i.e., $f_{\alpha} \in V^{\circ}$. Since $V^{\circ}$ is compact in $\mathcal{L}_{c} X$, by passing to further quasi-subnets of $\left(f_{\alpha}\right)$ and $\left(x_{\alpha}\right)$ we may assume $f_{\alpha} \stackrel{\text { c }}{\rightarrow} f$ for some $f$. As $\hat{x}_{\alpha} \stackrel{\text { c }}{\rightarrow} 0$ in $\mathcal{L}_{c} \mathcal{L}_{c} X$, we have $f_{\alpha}\left(x_{\alpha}\right)=\hat{x}_{\alpha}\left(f_{\alpha}\right) \rightarrow 0$ which contradicts $f_{\alpha}\left(x_{\alpha}\right) \geqslant 1$ for all $\alpha$.

The previous paragraph shows $j(X)$ is a (linear topological) subspace of $\mathcal{L}_{c} \mathcal{L}_{c} X$. We claim that it is dense. Assume towards a contradiction that $j(X)$ is not dense in $\mathcal{L}_{c} \mathcal{L}_{c} X$. An application of Hahn-Banach theorem gives a non-zero continuous linear functional on $\mathcal{L}_{c} \mathcal{L}_{c} X$ that 
vanishes on $j(X)$. Theorem 8.11 yields that the dual of $\mathcal{L}_{c} \mathcal{L}_{c} X$ is $\mathcal{L}_{c} X$. Hence, this separating functional is an element of $\mathcal{L}_{c} X$; denote it by $f$. Since $f$ vanishes on $j(X)$ implies $f(x)=(j(x))(f)=0$ for every $x \in X$, we obtain the contradiction $f=0$.

\section{ORder CONVERGENCE IN VECTOR LATTICES}

There are several convergences that are critically important in the theory of vector lattices, yet they are generally non-topological. These include order convergence, relative uniform convergence, and unbounded order convergence. While non-topological, these convergences fit in the framework of the theory of convergence structures. In this section, we present a survey of various properties of order convergence from the point of view of convergence structures. Throughout, $X$ will stand for a vector lattice and we only consider real scalars; most of the results extend easily to complex vector lattices. We refer the reader to AB06] for background and terminology of the theory of vector lattices and to AS05] for a discussion on definitions of order convergence.

Definition 9.1. A net $\left(x_{\alpha}\right)_{\alpha \in \Lambda}$ in a vector lattice $X$ is said to converge in order to some vector $x$ in $X$ if there exists a net $\left(u_{\gamma}\right)_{\gamma \in \Gamma}$ such that $u_{\gamma} \downarrow 0$ and for every $\gamma_{0} \in \Gamma$ there exists an $\alpha_{0} \in \Lambda$ such that $\left|x_{\alpha}-x\right| \leqslant u_{\gamma}$ for all $\alpha \geqslant \alpha_{0}$. We write $x_{\alpha} \stackrel{\circ}{\rightarrow} x$. Note that the net $\left(u_{\gamma}\right)_{\gamma \in \Gamma}$ may be indexed by a different index set than the original net. We say that $\left(u_{\gamma}\right)_{\gamma \in \Gamma}$ is a dominating net.

It is easy to see that order convergence satisfies (N1)-(N3), hence forms a convergence structure. We will denote this convergence space by $(X, \stackrel{\circ}{\rightarrow})$.

Definition 9.1 may be restated as follows: $x_{\alpha} \stackrel{\circ}{\rightarrow} x$ if there exists a net $\left(u_{\gamma}\right)$ such that $u_{\gamma} \downarrow 0$ and for every $\gamma$, the order interval $\left[x-u_{\gamma}, x+u_{\gamma}\right]$ contains a tail of $\left(x_{\alpha}\right)$. It is easy to see that a single net $\left(u_{\gamma}\right)$ may be replaced with two nets which "control" the difference from above and below:

Proposition 9.2. $x_{\alpha} \stackrel{\circ}{\rightarrow} x$ if there exist two nets $\left(a_{\gamma}\right)$ and $\left(b_{\gamma}\right)$ such that $a_{\gamma} \uparrow x, b_{\gamma} \downarrow x$, and for every $\gamma$, the order interval $\left[a_{\gamma}, b_{\gamma}\right]$ contains a tail of $\left(x_{\alpha}\right)$. 
This yields the following useful reformulation of the definition of order convergence:

Proposition 9.3. $x_{\alpha} \stackrel{\circ}{\rightarrow} x$ iff there is a nested decreasing net of order intervals such that the intersection of all these intervals is $\{x\}$ and each of the intervals contains a tail of $\left(x_{\alpha}\right)$.

Proposition 9.3 allows one to extend the definition of order convergence from vector lattices to partially ordered sets; cf. [Sch97, p. 171]. While some results of this section remain valid for partially ordered sets and ordered vector spaces, we will focus on vector lattices.

It is a standard easy fact that order limits are unique. In the language of convergence structures this precisely means that order convergence structure is Hausdorff. In vector lattice theory, a function $f: X \rightarrow Y$ between two vector lattices is said to be order continuous if $x_{\alpha} \stackrel{\circ}{\rightarrow} x$ implies $f\left(x_{\alpha}\right) \stackrel{\circ}{\rightarrow} f(x)$; this means that $f$ is continuous with respect to order convergence structures.

We will use the fact that if $\left(x_{\alpha}\right)$ is an increasing net then $x_{\alpha} \stackrel{\circ}{\rightarrow} x$ iff $x_{\alpha} \uparrow x$; the latter means $x=\sup x_{\alpha}$. Similarly, for a decreasing net we have $x_{\alpha} \stackrel{\mathrm{o}}{\rightarrow} x$ iff $x_{\alpha} \downarrow x$ iff $x=\inf x_{\alpha}$.

Is order convergence linear? It is easy to see from the definition that this convergence is translation invariant in the sense that $x_{\alpha} \stackrel{\circ}{\rightarrow} x$ implies $x_{\alpha}+a \stackrel{\circ}{\rightarrow} x+a$. This allows one to reduce many questions about order convergence to order convergence at zero. Moreover, it is a standard fact that addition is jointly continuous: if $\left(x_{\alpha}\right)_{\alpha \in A}$ converges in order to $x$ and $\left(y_{\beta}\right)_{\beta \in B}$ converges in order to $y$, the net $\left(x_{\alpha}+y_{\beta}\right)$ indexed by $A \times B$ converges in order to $x+y$. It is also easy to see that $x_{\alpha} \stackrel{\circ}{\rightarrow} x$ implies $\lambda x_{\alpha} \stackrel{\circ}{\rightarrow} \lambda x$ for every $\lambda \in \mathbb{R}$. The only outstanding issue is the joint continuity of scalar multiplication.

Proposition 9.4. A vector lattice is Archimedean iff its order convergence structure is linear.

Proof. By the preceding paragraph, we know that order convergence is linear iff scalar multiplication is jointly continuous. In the latter case $\frac{1}{n} u \stackrel{\circ}{\rightarrow} 0$ for every $u \in X_{+}$. This means $\frac{1}{n} u \downarrow 0$, so $X$ is Archimedean. 
Now suppose that $X$ is Archimedean. Let $\lambda_{\alpha} \rightarrow \lambda$ in $\mathbb{R}$ and $x_{\beta} \stackrel{\circ}{\rightarrow} x$ in $X$; we need to show $\lambda_{\alpha} x_{\beta} \stackrel{\circ}{\rightarrow} \lambda x$. By Remark 2.5 we may assume the two nets have the same index set. Passing to a tail, we may also assume $\left(\lambda_{\alpha}\right)$ is bounded; i.e., there is some $M>0$ where $\left|\lambda_{\alpha}\right| \leqslant M$ for all $\alpha$. Then

$$
\left|\lambda_{\alpha} x_{\alpha}-\lambda x\right| \leqslant M\left|x_{\alpha}-x\right|+\left|\lambda_{\alpha}-\lambda\right||x| \text {. }
$$

Clearly, $M\left|x_{\alpha}-x\right| \stackrel{\circ}{\rightarrow} 0$. Let $u_{n}=\frac{1}{n}|x|$. Since $X$ is Archimedean, $u_{n} \downarrow 0$. For every $n$, there exists $\alpha_{0}$ such that $\left|\lambda_{\alpha}-\lambda\right|<\frac{1}{n}$ for all $\alpha \geqslant \alpha_{0}$; it follows that $\left|\lambda_{\alpha}-\lambda\right||x| \leqslant u_{n}$ and, therefore, $\left|\lambda_{\alpha}-\lambda\right||x| \stackrel{\mathrm{o}}{\rightarrow} 0$. This yields $\lambda_{\alpha} x_{\alpha} \stackrel{\circ}{\rightarrow} \lambda x$.

The preceding proposition somewhat justifies the importance of the Archimedean Property and explains why most of the literature on vector lattices focuses on Archimedean spaces.

It is a standard fact from the theory of vector lattices that lattice operations $x \vee y, x \wedge y,|x|, x^{+}$, and $x^{-}$are order continuous. Indeed, the map $x \rightarrow|x|$ is order continuous because ||$x_{\alpha}|-| x|| \leqslant\left|x_{\alpha}-x\right|$. Similar arguments show that $x^{+}$and $x^{-}$are order continuous. It now follows from the fact that $x \vee y=x+(y-x)^{+}$that the map $(x, y) \mapsto x \vee y$ is jointly continuous. Finally, $x \wedge y=-((-x) \vee(-y))$ yields the joint continuity of the map $(x, y) \mapsto x \wedge y$.

Recall that a subset $A$ of a vector lattice is said to be order bounded when it is contained in an order interval. These sets correspond to the bounded sets of $(X, \stackrel{\circ}{\rightarrow})$ when order convergence is linear.

Proposition 9.5. A subset B in an Archimedean vector lattice is order bounded iff it is bounded in the order convergence structure.

Proof. Assume $A$ is order bounded: $A \subseteq[-u, u]$ for some $u \in X_{+}$. Let $\left(r_{\alpha}\right)_{\alpha \in \Lambda}$ be a net in $\mathbb{R}$ with $r_{\alpha} \rightarrow 0$. We need to show that $\left(r_{\alpha} a\right)$ converges in order to zero when viewed as a net over $\Lambda \times A$ directed by the first component. This follows from the fact that $\left|r_{\alpha} a\right| \leqslant\left|r_{\alpha}\right| u \stackrel{\circ}{\rightarrow} 0$.

Now suppose $A$ is bounded in $(X, \stackrel{\circ}{\rightarrow})$. Then the net $\left(\frac{1}{n} a\right)$ indexed by $\mathbb{N} \times A$ converges in order to zero. In particular, this net has an order bounded tail. It follows that there exists $u \in X_{+}$and $n_{0} \in \mathbb{N}$ such that $\frac{1}{n_{0}} a \in[-u, u]$ for all $a \in A$. Then $A \subseteq\left[-n_{0} u, n_{0} u\right]$. 
It is easy to see that every order convergent net has an order bounded tail. In the language of convergence structures this translates to the following.

Corollary 9.6. If $X$ is an Archimedean vector lattice then the convergence space $(X, \stackrel{\circ}{\rightarrow})$ is locally bounded.

Example 9.7. Let $X=\mathbb{R}^{2}$ with the lexicographic order. Note that $X$ is non-Archimedean and its order convergence structure is not linear. Let $A$ be the $y$-axis. Clearly, $A$ is order bounded as $A \subseteq[-u, u]$ for $u=(1,0)$. However, $A$ is not bounded in the natural convergence structure of $\mathbb{R}^{2}$. This example illustrates that in the absence of linearity, the concept of order boundedness may be "ill behaved".

Sublattices. Let $Y$ be a sublattice of an Archimedean vector lattice $X$. In particular, $Y$ is a vector lattice in its own right. It is well known that order convergence in $Y$ need not agree with the order convergence inherited from $X$. In other words, order convergence generally depends on the ambient space. For example, the unit vector basis $\left(e_{n}\right)$ in $c_{0}$ fails to converge in order (it is not even order bounded) but, viewed as a sequence in $\ell_{\infty}$, it converges in order to zero. It may also happen that $x_{\alpha} \stackrel{\circ}{\rightarrow} 0$ in $Y$ but not in $X$; see, e.g., Example 2.6 in GXT17. In particular, a sublattice need not be a subspace in the sense of convergence structures.

$Y$ is said to be a regular sublattice of $X$ if $y_{\alpha} \stackrel{\circ}{\rightarrow} 0$ in $Y$ implies $y_{\alpha} \stackrel{\circ}{\rightarrow} 0$ in $X$. Equivalently, the inclusion map is order continuous.

A sublattice $T$ is said to be majorizing in $X$ if for every $x \in X_{+}$ there exists $y \in Y_{+}$such that $x \leqslant y$. We say that $Y$ is order dense in $X$ if for every non-zero $x \in X_{+}$there exists $y \in Y_{+}$such that $0<y \leqslant x$. Theorem 1.23 in [AB03 asserts that every order dense sublattice is regular. In particular, every Archimedean vector lattice $X$ is order dense and, therefore, regular in its order (or Dedekind) completion $X^{\delta}$.

One may ask whether being order dense is the same as being dense with respect to the order convergence structure; that is, whether order dense is equivalent to every $x \in X$ is an order limit of some net in $Y$. It is shown in Theorem 1.27 of $\mathrm{AB} 03$ that $Y$ is order dense iff 
$a=\sup [0, a] \cap Y$ for every $a \in X_{+}$where the supremum is evaluated in $X$. It follows that if $Y$ is order dense then it is dense with respect to the order convergence structure. Example 2.6 in GXT17 shows that the converse is generally false. Thus, there is an unfortunate clash of terminologies: a sublattice which is dense in the sense of order convergence structure need not be order dense. However, Corollary 2.13 of GXT17] shows that the two concepts of density agree for regular sublattices. In particular, every Archimedean vector lattice $X$ is dense in $X^{\delta}$ in the sense of the order convergence structure.

Theorem 2.8 of GXT17 asserts that if $Y$ is order dense and majorizing, and $\left(y_{\alpha}\right)$ is a net in $Y$ then $y_{\alpha} \stackrel{\circ}{\rightarrow} 0$ in $Y$ iff $y_{\alpha} \stackrel{\circ}{\rightarrow} 0$ in $X$. That is, $Y$ is a convergence subspace of $X$. A special case of this is the following fact that was established in AS05]:

Theorem 9.8. Every Archimedean vector lattice $X$ is a convergence subspace in its order completion $X^{\delta}$; i.e., $x_{\alpha} \stackrel{\mathrm{o}}{\rightarrow} 0$ in $X$ iff $x_{\alpha} \stackrel{\mathrm{o}}{\rightarrow} 0$ in $X^{\delta}$ for every net $\left(x_{\alpha}\right)$ in $X$.

It is also worth mentioning that Corollary 2.12 in GXT17] says that if $Y$ is a regular sublattice of $X$ then the order convergences of $X$ and of $Y$ agree for order bounded nets.

Order completeness. Recall that a linear convergence structure is complete when every Cauchy net is convergent. A vector lattice $X$ is said to be order (or Dedekind) complete if every bounded above increasing net has a supremum. We are going to show that an Archimedean vector lattice is order complete iff its order convergence structure is complete; cf. Proposition 2.3 in [GXT17.

Let $X$ be an order complete vector lattice and $\left(x_{\alpha}\right)$ an order bounded net in $X$. It is a standard exercise that $x_{\alpha} \stackrel{\circ}{\rightarrow} x$ iff $x=\inf _{\alpha} \sup _{\beta \geqslant \alpha} x_{\beta}=$ $\sup _{\alpha} \inf _{\beta \geqslant \alpha} x_{\beta}$. It follows that in Proposition 9.2 we can take $a_{\alpha}=$ $\inf _{\beta \geqslant \alpha} x_{\beta}$ and $b_{\alpha}=\sup _{\beta \geqslant \alpha} x_{\beta}$. In particular, this means that the "control" nets may be chosen over the same index set as the original net. Then the dominating net in Definition 9.1 may also be chosen over the same index set as $\left(x_{\alpha}\right)$. Thus, in this setting we obtain $x_{\alpha} \stackrel{\circ}{\rightarrow} x$ iff there exists a net $\left(u_{\alpha}\right)$ over the same index where $u_{\alpha} \downarrow 0$ and $\left|x_{\alpha}-x\right| \leqslant u_{\alpha}$ for every $\alpha$. 
A net $\left(x_{\alpha}\right)_{\alpha \in \Lambda}$ in a vector lattice $X$ is said to be order Cauchy if it is Cauchy in the order convergence structure; i.e., if the net $\left(x_{\alpha}-x_{\beta}\right)_{(\alpha, \beta)}$ is order null. The latter net is indexed by $\Lambda \times \Lambda$ under the componentwise order.

Lemma 9.9. Every monotone order bounded net in an Archimedean vector lattice is order Cauchy.

Proof. Suppose that $x_{\alpha} \uparrow \leqslant u$ in $X$. Then $z:=\sup x_{\alpha}$ exists in $X^{\delta}$. It follows that the double net $\left(x_{\alpha}-x_{\beta}\right)_{(\alpha, \beta)}$ is order null in $X^{\delta}$ and, therefore, in $X$. The case of a decreasing net may be handled in a similar way.

Proposition 9.10. An Archimedean vector lattice is order complete iff every order Cauchy net is order convergent.

Proof. Let $\left(x_{\alpha}\right)$ be an order Cauchy net in an order complete vector lattice; we will show that it is order convergent. It is easy to see that $\left(x_{\alpha}\right)$ has an order bounded tail, so we may assume that $\left(x_{\alpha}\right)$ is order bounded. Put

$$
x=\sup a_{\alpha} \text { where } a_{\alpha}=\inf _{\beta \geqslant \alpha} x_{\beta} \text {, and let } y=\inf b_{\alpha} \text { where } b_{\alpha}=\sup _{\beta \geqslant \alpha} x_{\beta} .
$$

Then $a_{\alpha} \leqslant x \leqslant y \leqslant b_{\alpha}$ for every $\alpha$. By the preceding argument, it suffices to show that $x=y$. Let $\left(v_{\alpha, \beta}\right)$ be a net such that $v_{\alpha, \beta} \downarrow 0$ and $\left|x_{\alpha}-x_{\beta}\right| \leqslant v_{\alpha, \beta}$. Fix a pair $\left(\alpha_{0}, \beta_{0}\right)$. Let $\alpha$ be such that $\alpha \geqslant \alpha_{0}$ and $\alpha \geqslant \beta_{0}$. For every $\beta$ with $\beta \geqslant \alpha$, we have $(\alpha, \beta) \geqslant\left(\alpha_{0}, \beta_{0}\right)$, so that $\left|x_{\alpha}-x_{\beta}\right| \leqslant v_{\alpha_{0}, \beta_{0}}$. It follows that $x_{\beta} \in\left[x_{\alpha}-v_{\alpha_{0}, \beta_{0}}, x_{\alpha}+v_{\alpha_{0}, \beta_{0}}\right]$, which yields $a_{\alpha}, b_{\alpha} \in\left[x_{\alpha}-v_{\alpha_{0}, \beta_{0}}, x_{\alpha}+v_{\alpha_{0}, \beta_{0}}\right]$. We thus obtain $0 \leqslant b_{\alpha}-a_{\alpha} \leqslant$ $2 v_{\alpha_{0}, \beta_{0}}$ and so $0 \leqslant y-x \leqslant 2 v_{\alpha_{0}, \beta_{0}}$. If follows that $x-y=0$.

Conversely, suppose that every order Cauchy net in $X$ is order convergent. Let $0 \leqslant x_{\alpha} \uparrow \leqslant u$. By the lemma, $\left(x_{\alpha}\right)$ is order Cauchy, hence order convergent; it follows that $\sup x_{\alpha}$ exists.

Recall that the most common construction of the order completion of an Archimedean vector lattice is analogous to the construction of $\mathbb{R}$ using Dedekind cuts; see, for example, [Vul67]. Also recall the alternate construction of $\mathbb{R}$ using equivalence classes of Cauchy sequences of rationals. 
The latter construction has an analogue for Archimedean vector lattices. Given an Archimedean vector lattice $X$, we may view the order convergence structure of its order completion $X^{\delta}$ as a Cauchy completion of the order convergence structure on $X$ in the following way: $X$ is a dense subspace of $X^{\delta}$ in the sense of convergence structures, and the elements of $X^{\delta}$ are limits of order Cauchy nets in $X$. To see this, note that for every $x \in X^{\delta}$ there exists a net $\left(x_{\alpha}\right)$ in $X$ with $x_{\alpha} \stackrel{\circ}{\rightarrow} x$ in $X^{\delta}$. It follows that $\left(x_{\alpha}\right)$ is order Cauchy in $X^{\delta}$ and, therefore, in $X$ by Proposition 9.8. Conversely, every order Cauchy net in $X$ remains order Cauchy in $X^{\delta}$ by Proposition 9.8, the previous proposition now yields that it converges in order in $X^{\delta}$.

Filter considerations. Proposition 9.3 yields a filter version of order convergence: a filter $\mathcal{F}$ on a vector lattice $X$ converges in order to $x$ (written $\mathcal{F} \stackrel{\circ}{\rightarrow} x$ ) if $\mathcal{F}$ contains a nested decreasing family of order intervals whose intersection is $\{x\}$; see [Sch74, p. 54] or [AW05]. It can now be easily verified that this filter convergence structure corresponds exactly to the net convergence structure in Definition 9.1. This allows one to use the full power of the theory of filter convergence structures when dealing with order convergence.

In particular, we can discuss the concepts of local convexity and regularity from the theory of filter convergence structures. A convergence space is regular when $\mathcal{F} \rightarrow x$ implies that the filter generated by the closures of the sets in $\mathcal{F}$ still converges to $x$; this is not the same as a regular sublattice. A convergence vector space is locally convex if $\mathcal{F} \rightarrow x$ implies the filter generated by the convex hulls of the sets in $\mathcal{F}$ still converges to $x$. Now let $X$ be a vector lattice equipped with its (filter) order convergence structure. Since order intervals are order closed and convex, we immediately obtain the following results.

Proposition 9.11. The order convergence structure is regular and locally convex. 


\section{Relative UNIFORM CONVERGENCE AND ORDER TOPOLOGY}

We will show in this section that the Mackey modification of order convergence is relative uniform convergence. We then discuss the topological modification of order convergence and characterize the spaces where order convergence is topological.

Relative uniform convergence. Let $X$ be a vector lattice. We say that a net $\left(x_{\alpha}\right)$ converges to some $x \in X$ (relatively) uniformly and write $x_{\alpha} \stackrel{\mathrm{u}}{\rightarrow} x$ if there exists $e \in X_{+}$such that for every $\varepsilon>0$ there exists $\alpha_{0}$ such that $\left|x_{\alpha}-x\right| \leqslant \varepsilon e$ for all $\alpha \geqslant \alpha_{0}$.

Let $e \in X_{+}$. For $x \in X$, put

$$
\|x\|_{e}=\inf \{\lambda \geqslant 0:|x| \leqslant \lambda e\} .
$$

It is clear that $\|x\|_{e}<\infty$ iff $x$ is in the principal ideal $I_{e}$ of $e$. It is also easy to see that $\|\cdot\|_{e}$ is a lattice seminorm on $I_{e}$; it is a norm when $X$ is Archimedean. Then $x_{\alpha} \stackrel{\mathrm{u}}{\rightarrow} x$ iff $\left\|x_{\alpha}-x\right\|_{e} \rightarrow 0$ for some $e \in X_{+}$.

If $X$ is an Archimedean vector lattice then relative uniform convergence is a linear Hausdorff convergence structure on $X$. This may be verified directly. It also follows immediately from the next result:

Theorem 10.1. Let $X$ be an Archimedean vector lattice. Then its relative uniform convergence structure is the Mackey modification of its order convergence structure.

Proof. Recall that by Proposition 9.5, order bounded sets in $X$ are the sets that are bounded in the order convergence structure. The result now follows easily from the fact that a set is order bounded iff it is contained in $[-e, e]$ for some $e \in X_{+}$.

The sequential variant of this theorem was proved in [VdW16].

Corollary 10.2. Let $\mathcal{F}$ be a filter in an Archimedean vector lattice. Then $\mathcal{F} \stackrel{\mathrm{u}}{\rightarrow} 0$ iff there exists $e \in X_{+}$such that $\left[-\frac{1}{n} e, \frac{1}{n} e\right] \in \mathcal{F}$ for every $n \in \mathbb{N}$.

The following result characterizes order boundedness of an operator as a form of continuity. This was proved in Theorem 5.1 of [TT20], but now it follows immediately from Theorem 10.1 and Proposition 3.9. 
Theorem 10.3. Let $T: X \rightarrow Y$ be a linear operator between Archimedean vector lattices. The following are equivalent:

(i) $T$ is order bounded; that is, $T$ maps order bounded sets to order bounded sets.

(ii) $T$ is relative uniformly continuous: $x_{\alpha} \stackrel{\mathrm{u}}{\rightarrow} x$ implies $T x_{\alpha} \stackrel{\mathrm{u}}{\rightarrow} T x$.

(iii) $T$ is relative uniformly-to-order continuous; that is, $x_{\alpha} \stackrel{\mathrm{u}}{\rightarrow} x$ implies $T x_{\alpha} \stackrel{\circ}{\rightarrow} T x$.

It is also observed in [TT20] that a Banach lattice is order continuous iff order convergence and relative uniform convergence structures agree iff order convergence and relative uniform convergence agree on sequences. Thus, the order convergence structure on Banach lattice $X$ is Mackey iff $X$ is order continuous. Combining this with the preceding theorem, we get the following.

Corollary 10.4. A linear operator from an order continuous Banach lattice to an Archimedean vector lattice is order bounded iff it is order continuous.

In the filter language, it is easy to see that $\mathcal{F} \stackrel{u}{\rightarrow} 0$ iff there exists $u \in X_{+}$such that $\mathcal{N}_{0}[-u, u] \subseteq \mathcal{F}$. Here, as before, $\mathcal{N}_{0}$ stands for the neighborhood filter of zero in $\mathbb{R}$. Let $\mathcal{G}$ be the filter generated by $\mathcal{N}_{0}[-u, u]$; it is easy to see that the order intervals $\left[-\frac{1}{n} u, \frac{1}{n} u\right]$ as $n \in \mathbb{N}$ form a base of $\mathcal{G}$. This proves the following:

Proposition 10.5. Relative uniform convergence of an Archimedean vector lattice is first countable.

It was also observed in TT20] that if $Y$ is a relative uniformly closed sublattice of a relative uniformly complete vector lattice $X$ and $\left(x_{k}\right)$ is a sequence in $Y$, then $x_{k} \stackrel{\text { u }}{\rightarrow} 0$ in $Y$ iff $x_{k} \stackrel{\text { u }}{\rightarrow} 0$ in $X$.

Order Convergence and Topology. As we mentioned in the beginning of the paper, Ordman observed in Ord66] that almost everywhere convergence of measurable functions is not topological. The argument is very simple; we reproduce it here for the convenience of the reader:

Example 10.6. Consider the space $L_{0}[0,1]$ of all Lebesgue measurable functions on $[0,1]$ equipped with convergence almost everywhere. Let 
$s_{0}=0$ and $s_{n}=\sum_{k=1}^{n} \frac{1}{k}$ for $n \in \mathbb{N}$, then put $A_{n}=\left[s_{n-1}, s_{n}\right] \bmod 1$. Then $A_{n} \subseteq[0,1]$ and the Lebesgue measure of $A_{n}$ is $\frac{1}{n}$. The sequence $\left(x_{n}\right)$ defined by $x_{n}=\mathbb{1}_{A_{n}}$ is called the typewriter sequence. It is easy to see that every subsequence of $\left(x_{n}\right)$ has a further subsequence that converges to zero. If the convergence were topological, we would have $x_{n} \rightarrow 0$, but the latter is false.

For sequences in $L_{0}[0,1]$, order convergence agrees with convergence almost everywhere, hence the typewriter sequence from the example shows that order convergence is generally not topological. We will show that order convergence may be very far from being topological; it is topological iff the space is finite-dimensional. Moreover, its topological modification is the order topology, which may be very coarse: it need not be linear or Hausdorff.

In vector lattice theory, a subset $A$ of a vector lattice is said to be order closed if $x_{\alpha} \stackrel{\circ}{\rightarrow} x$ and $\left\{x_{\alpha}\right\} \subseteq A$ imply $x \in A$; this agrees with the definition of closed sets in the order convergence structure. The collection of all order closed sets forms a topology; following [LZ73, p. 80], we call it the order topology. In the language of convergence structures, this is precisely the topological modification of the order convergence structure; we will denote this topology by $\tau_{o}$. By Proposition 3.5. $\tau_{o}$ is the finest topology on $X$ whose convergence is weaker than order convergence.

It is easy to see that the order topology is translation invariant, and it follows immediately from the definition that points are closed. By Theorem 5.1 on pp. 34-35 of [KN76], a topological vector space is Hausdorff iff points are closed. Therefore, if $\tau_{o}$ is linear, it is automatically Hausdorff. The following example shows the order topology need not be Hausdorff or linear; cf. [Vla69, p. 146].

Example 10.7. Order topology on $C[0,1]$ is not Hausdorff and not linear. Suppose it is Hausdorff. Let $U$ and $V$ be open neighbourhoods of 0 and of $\mathbb{1}$, respectively, with $U \cap V=\varnothing$. Let $\left(t_{k}\right)$ be an enumeration of the rational numbers in $(0,1)$. For each $k, n \in \mathbb{N}$, let $x_{k, n}$ be the continuous function such that $x_{k, n}\left(t_{k}\right)=1, x_{n, k}$ vanishes outside of $\left[t_{k}-\frac{1}{n}, t_{k}+\frac{1}{n}\right]$, and is linear on $\left[t_{k}-\frac{1}{n}, t_{k}\right]$ and on $\left[t_{k}, t_{k}+\frac{1}{n}\right]$. For 
each fixed $k$, we clearly have $x_{k, n} \downarrow 0$ as $n \rightarrow \infty$. It follows that $x_{k, n} \stackrel{\circ}{\rightarrow} 0$ and, therefore, $x_{k, n} \stackrel{\tau_{o}}{\rightarrow} x$ as $n \rightarrow \infty$. Choose $n_{1}$ such that $y_{1}:=x_{1, n_{1}} \in U$. Next we observe that $y_{1} \vee x_{2, n} \downarrow y_{1}$. It follows from $y_{1} \in U$ that $y_{2}:=y_{1} \vee x_{2, n_{2}} \in U$ for some $n_{2}$. Iterating this process, we construct a sequences $\left(n_{k}\right)$ in $\mathbb{N}$ and $\left(y_{k}\right)$ in $U$ such that $y_{k}=y_{k-1} \vee x_{k, n_{k}}$ for every $k>1$. It follows that $y_{k} \uparrow$ and $y_{k}\left(t_{i}\right)=1$ as $i=1, \ldots, k$. This yields $y_{k} \uparrow \mathbb{1}$. Therefore, there exists $k_{0}$ such that $y_{k_{0}} \in V$, which contradicts $U$ and $V$ being disjoint.

So the order topology on $C[0,1]$ can fail to be Hausdorff and, therefore, is not generally a linear topology.

While the preceding example shows that the order topology may be rather poor, there are situations where it is "nice". Let $X$ be Banach lattice. It is well known that every norm convergent sequence in $X$ has a subsequence that converges in order to the same limit. It follows that every order closed set in $X$ is norm closed: so the norm topology on $X$ is stronger than its order topology. If, in addition, $X$ is order continuous, a set is norm closed iff it is order closed. Thus, the topological modification of order convergence agrees with the norm topology in order continuous Banach lattices.

We now provide a useful characterization of order neighbourhoods, which are sometimes called net-catching sets in the literature.

Proposition 10.8. Let $U$ be a subset of a vector lattice $X$ and $x \in X$. The following are equivalent:

(i) $U$ is an order neighborhood of $x$;

(ii) if $u_{\alpha} \downarrow 0$ then there exists $\alpha_{0}$ such that $\left[x-u_{\alpha}, x+u_{\alpha}\right] \subseteq U$ for all $\alpha \geqslant \alpha_{0}$.

Proof. Without loss of generality we may assume $x=0$.

(ii) $\Rightarrow$ (iii) Suppose that $U$ satisfies (ii) but fails (iii). Then there exists a net $\left(u_{\alpha}\right)$ such that $u_{\alpha} \downarrow 0$ but for every $\alpha$, the interval $\left[-u_{\alpha}, u_{\alpha}\right]$ is not entirely contained in $U$. We can then find $x_{\alpha}$ in this interval such that $x_{\alpha} \notin U$. This results in a net $\left(x_{\alpha}\right)$ such that $x_{\alpha} \stackrel{\circ}{\rightarrow} 0$, yet no $x_{\alpha}$ is in $U$; a contradiction.

(iii) $\Rightarrow$ (ii) Suppose that $\left(x_{\alpha}\right)_{\alpha \in \Lambda} \rightarrow 0$. Let $\left(u_{\gamma}\right)_{\gamma \in \Gamma}$ be a dominating net as in Definition 9.1. By assumption, there exists $\gamma_{0}$ such that 
$\left[-u_{\gamma_{0}}, u_{\gamma_{0}}\right] \subseteq U$. On the other hand, a tail of $\left(x_{\alpha}\right)$ is contained in $\left[-u_{\gamma_{0}}, u_{\gamma_{0}}\right]$, and, therefore in $U$.

It was shown in DEM20 that order convergence is topological precisely in finite-dimensional spaces. We will now provide a short proof of this fact.

Lemma 10.9. Let $\tau$ be a linear topology on a vector lattice $X$. If $\tau$ convergence is stronger than order convergence then $X$ is Archimedean, has a strong unit $e$, and $x_{\alpha} \stackrel{\tau}{\rightarrow} 0$ implies $\left\|x_{\alpha}\right\|_{e} \rightarrow 0$.

Proof. To show that $X$ is Archimedean, suppose $0 \leqslant x \leqslant \frac{1}{n} u$ for all $n \in \mathbb{N}$. Since scalar multiplication is $\tau$-continuous, we have $\frac{1}{n} u \stackrel{\tau}{\rightarrow} 0$, which yields $\frac{1}{n} u \stackrel{\circ}{\rightarrow} 0$ and, therefore, $x=0$.

Let $\mathcal{N}_{0}$ be the filter of zero neighborhoods for $\tau$. Then we have $\mathcal{N}_{0} \stackrel{\tau}{\rightarrow} 0$ and, therefore, $\mathcal{N}_{0} \stackrel{\circ}{\rightarrow} 0$. Since order convergence is locally bounded by Corollary 9.6, $\mathcal{N}_{0}$ contains an order bounded member. That is, there exists a $U \in \mathcal{N}_{0}$ and $e \in X_{+}$such that $U \subseteq[-e, e]$. It follows that $e$ is a strong unit.

To prove the last claim, suppose that $x_{\alpha} \stackrel{\tau}{\rightarrow} 0$ for some net $\left(x_{\alpha}\right)$. Fix $\varepsilon>0$ and let $U$ be as above. Then $\varepsilon U \in \mathcal{N}_{0}$ and there exists $\alpha_{0}$ such that $x_{\alpha} \in \varepsilon U$ whenever $\alpha \geqslant \alpha_{0}$. It follows that $\left|x_{\alpha}\right| \leqslant \varepsilon e$; i.e., $\left\|x_{\alpha}\right\|_{e} \leqslant \varepsilon$.

Theorem 10.10. Order convergence on a vector lattice $X$ arises from a linear topology iff $X$ is finite-dimensional and Archimedean.

Proof. Let $X$ be a finite-dimensional Archimedean vector lattice. Then $X$ is lattice-isomorphic to $\mathbb{R}^{n}$ for some $n$. So, without loss of generality, we assume $X=\mathbb{R}^{n}$. Then order convergence agrees with the coordinate-wise convergence, which is topological.

Suppose that there is a linear topology $\tau$ on $X$ with $x_{\alpha} \stackrel{\tau}{\rightarrow} 0$ iff $x_{\alpha} \stackrel{\circ}{\rightarrow} 0$ for every net $\left(x_{\alpha}\right)$ in $X$. By Theorem $10.9 X$ is Archimedean, has a strong unit $e$, and $x_{\alpha} \stackrel{\tau}{\rightarrow} 0$ implies $\left\|x_{\alpha}\right\|_{e} \rightarrow 0$. For the sake of contradiction, assume that $X$ is infinite-dimensional. Then $X$ contains an infinite disjoint sequence of non-zero vectors, say, $\left(x_{n}\right)$. Without loss of generality, we may assume $\left\|x_{n}\right\|_{e}=1$ for each $n$. Viewed as an order bounded disjoint sequence in $X^{\delta},\left(x_{n}\right)$ is order null in $X^{\delta}$. Then 
$x_{n} \stackrel{\circ}{\rightarrow} 0$ in $X$ by Theorem 9.8 . Our assumption gives $x_{n} \stackrel{\tau}{\rightarrow} 0$; hence $\left\|x_{n}\right\|_{e} \rightarrow 0$ by Lemma 10.9, a contradiction.

Corollary 10.11. Order convergence on an Archimedean vector lattice $X$ is topological iff $X$ is finite-dimensional.

Proof. Suppose that order convergence on $X$ comes from a topology $\tau$. Since addition and scalar multiplication are jointly continuous with respect to order convergence, it follows that $\tau$ is linear. Now apply Theorem 10.10.

Example 10.12. Consider $X=\mathbb{R}^{2}$ with the lexicographic order. Note that $X$ fails the Archimedean Property. The sequence $\left(\frac{1}{n}, 0\right)$ converges to zero in every Hausdorff linear topology on $X$, yet it does not converge to zero in order.

Proposition 10.13. Relative uniform convergence on an Archimedean vector lattice $X$ is topological iff $X$ has a strong unit.

Proof. If relative uniform convergence arises from a topology, then $X$ has a strong unit by Lemma 10.9. Conversely, if $X$ has a strong unit, say, $e$, then relative uniform convergence agrees with convergence in $\|\cdot\|_{e^{-n o r m} \text {. }}$

Remark 10.14. It was observed in Proposition 3.1.3 of [BB02] that a convergence vector space is topological iff it is pretopological. This implies that "topological" may be replaced with "pretopological" in Corollary 10.11 and Proposition 10.13. In particular, in an infinitedimensional Archimedean vector lattice, an arbitrary mixing of order (or uniformly) convergent nets may spoil the convergence.

In the last few years, several variants of unbounded convergences came to prominence in vector lattice theory. These provide more examples of non-topological convergence structures. We will discuss unbounded convergences from the perspective of the theory of convergence structures in a separate paper.

Acknowledgements. The authors would like to thank M. Taylor for valuable discussions. 


\section{REFERENCES}

[AA72] J.F. Aarnes and P.R. Andenaes, On nets and filters, Math. Scand. 31 (1972), 285-292.

[AS05] Y. Abramovich and G. Sirotkin, On order convergence of nets, Positivity, 9, 2005, 287-292.

[AB03] C.D. Aliprantis and O. Burkinshaw, Locally solid Riesz spaces with applications to economics, 2nd ed., AMS, Providence, RI, 2003.

[AB06] C.D. Aliprantis and O. Burkinshaw, Positive operators, 2nd edition, Springer, 2006.

[AW05] R. Anguelov and J.H. van der Walt, Order convergence structure on C(X). Quaest. Math. 28 (2005), no. 4, 425-457.

[Ars77] Z. Artstein, Continuous dependence of solutions of operator equations. I, Trans. Amer. Math. Soc. 231 (1977), no. 1, 143-166.

[AEG] A. Aydın, E. Emelyanov, S. Gorokhova, Full Lattice Convergence on Riesz Spaces, preprint arXiv:2004.04879v1 [math.FA]

[BB02] R. Beattie and H.-P. Butzmann, Convergence Structures and Applications to Functional Analysis, Kluwer Academic Publishers, Dordrecht, 2002.

[DEM20] Y.A. Dabboorasad, E.Y. Emelyanov and M.A.A. Marabeh, Order Convergence in infinite-dimensional vector lattices is not topological, $U z b$. Mat. Zh. (2020)(1) 159-166.

[GXT17] N. Gao, F. Xanthos and V.G. Troitsky, Uo-convergence and its applications to Cesàro means in Banach lattices, Israel J. Math., 220, 2017, 649-689.

[HZW10] W.K. Ho, D. Zhao, and W.S. Wee, D-Completions of Net Convergence Structures, in Quantitative Logic and Soft Computing, 2010, AISC 82, pp. 93-110.

[Kat67] M. Katětov, Convergence structures, General Topology and its Relations to Modern Analysis and Algebra, Praha Academia Publishing, 1967, 207-216.

[Kel55] J.L. Kelley, General topology, D. Van Nostrand Company, Inc., TorontoNew York-London, 1955

[KN76] J.L. Kelley and I. Namioka, Linear topological spaces, Second corrected printing. Graduate Texts in Mathematics, No. 36. Springer-Verlag, New York-Heidelberg, 1976.

[LZ73] W.A.J. Luxemburg and A.C. Zaanen, Riesz spaces. Vol. I NorthHolland Publishing Co., Amsterdam-London, 1971.

[OBr21] M. O'Brien, A Theory of Net Convergence with Applications to Vector Lattices, Ph.D. Thesis, University of Alberta, 2021 (in preparation). 
[Ord66] E.T. Ordman, Convergence almost everywhere is not topological, Amer. Math. Monthly, 73(2), 1966, 182-183.

[Pea88] B.J. Pearson, Spaces defined by convergence classes of nets, Glas. Mat. Ser. III 23(43) (1988), no. 1, 135-142.

[Sch74] H.H. Schaefer, Banach lattices and positive operators, Springer-Verlag, Berlin, 1974.

[Sch97] E. Schechter, Handbook of analysis and its foundations, Academic Press, Inc., San Diego, CA, 1997.

[TT20] M.A. Taylor and V.G. Troitsky, Bibasic sequences in Banach lattices. J. Funct. Anal., 278(10), 2020, 108448.

[VdW06] J.H. Van der Walt, Order convergence on Archimedean vector lattices and applications, Ph.D. Thesis, University of Pretoria, 2006.

[VdW11] J.H. Van der Walt, The order convergence structure, Indag. Math. (N.S.), 21, 2011, 138-155.

[VdW16] J.H. Van der Walt, A closed graph theorem for order bounded operators, Quaestiones Math. 39(2) 2016, 167-178.

[Vla69] D.A. Vladimirov, Boolean algebras, Nauka, Moscow, 1969.

[vIm12] H. van Imhoff, Order-convergence in partially ordered vector spaces, Master's Thesis, Mathematisch Instituut, Universiteit Leiden, 2012.

[Vul67] B.Z. Vulikh, Introduction to the theory of partially ordered spaces (translated from the Russian), Wolters-Noordhoff, Groningen, 1967,

[Wil70] S. Willard, General topology, Addison-Wesley Publishing Co., Reading, Mass.-London-Don Mills, Ont., 1970.

Email address: mjbeauli@ualberta.ca

Email address: troitsky@ualberta.ca

Department of Mathematical and Statistical Sciences, University of Alberta, Edmonton, AB, T6G 2G1, Canada.

Email address: janharm.vanderwalt@up.ac.za

Department of Mathematics and Applied Mathematics, University of Pretoria, Corner of Lynnwood Road and Roper Street, Hatfield 0083, Pretoria, South Africa 